\title{
Towards the Noise Reduction of Piezoelectrical-Driven Synthetic Jet Actuators
}

\author{
Mark Jabbal ${ }^{*}$ and Stephanos Kykkotis ${ }^{\dagger}$ \\ Brunel University, London, UB8 3PH, United Kingdom
}

\begin{abstract}
This paper details an experimental investigation aimed at reducing the noise output of piezoelectrical-driven synthetic jet actuators without compromising peak jet velocity. Specifically, the study considers double-chamber ('back-to-back') actuators for anti-phase noise suppression and corrugated-lobed orifices as a method to enhance turbulent mixing of the jets to suppress jet noise. The study involved the design, manufacture and bench test of interchangeable actuator hardware. Hot-wire anemometry and microphone recordings were employed to acquire velocity and noise measurements respectively for each chamber configuration and orifice plate across a range of excitation frequencies and for a fixed input voltage. The data analysis indicated a $32 \%$ noise reduction (20 dBA) from operating a singlechamber, circular orifice SJA to a double-chamber, corrugated-lobed orifice SJA at the Helmholtz resonant frequency. Results also showed there was a small reduction in peak jet velocity of $7 \%(\sim 3 \mathrm{~m} / \mathrm{s})$ between these two cases based on orifices of the same discharge area. Finally, the electrical-to-fluidic power conversion efficiency of the double-chamber actuator was found to be $15 \%$ across all orifice designs at the resonant frequency; approximately double the efficiency of a single-chamber actuator. This work has thus demonstrated feasible gains in noise reduction and power efficiency through synthetic jet actuator design.
\end{abstract}

\section{Nomenclature}

$=$ orifice area

$=$ speed of sound

$=$ piezoceramic capacitance

$=$ chamber diameter

$=$ orifice diameter

$=$ elastic modulus; electrical power

$=$ fluidic power

$=$ actuation frequency

$=$ chamber height

$=$ orifice height

$=$ peak current

$=$ non-dimensional frequency parameter

$=$ actuator energy loss

$=$ diaphragm radius

$=$ instantaneous time; diaphragm thickness

$=$ velocity

$=$ peak-to-peak voltage

$=$ peak voltage

$=$ phase difference

$=$ electrical-to-fluidic power efficiency

$=$ Poisson ratio

$=$ air density

$=$ damping coefficient

\footnotetext{
* Lecturer/Assistant Professor, School of Engineering \& Design, UB8 3PH, MAIAA, mark_jabbal@ hotmail.com

${ }^{\dagger}$ Bachelor of Engineering Graduate, School of Engineering \& Design, UB8 3PH
} 


\section{Introduction}

TSSUES concerning the sustainability of aviation, amidst rapid growth in air traffic and moderate gains in aircraft efficiency, has led to the aerospace sector being set stringent goals to reduce its environmental impact. To reflect the importance of these issues from a European perspective, the European Commission has set the target within the Flight Path 2050 Vision of reducing CO2 and noise emissions of an aircraft by $80 \%$ and $65 \%$ respectively ${ }^{1}$.

Active flow control represents one means through which aircraft efficiency could be improved via aerodynamics, with synthetic jet actuators (SJAs) as a potential candidate technology.

A SJA is a zero-net mass flux device which produces a non-zero momentum output and thus negates the need for a network of pneumatic ducts. Instead, it generates fluidic power through an orifice on one side of a chamber using an oscillating diaphragm on the opposite side. In the case of a piezoceramic diaphragm, an input electrical supply is required to create the oscillatory motion. The momentum that a SJA induces into a fluid flow allows for delay in boundary layer separation, which could be used to improve the effectiveness of aircraft high-lift devices. Compared to other active flow control devices, a SJA allows for more flexibility with its relatively compact structure and with its ability to be controlled independently from its neighbouring actuators in an array. It has also been demonstrated that SJAs have the advantage of a greater spreading rate than that of an continuous jet of the same time-averaged jet velocity, thereby yielding a greater region of influence on the working domain.

One of the prime limitations of a SJA is its significantly high noise output generated from the motion of the diaphragm and high velocity jet stream mixing with the atmosphere. It is important that SJAs have no adverse impact on aircraft noise to comply with industry efforts to move towards more silent aircraft, in addition to a reduction in environmentally deleterious emissions which may be brought about through improved aerodynamics by flow control. Unfortunately SJA effectiveness often dictates operation at the actuator resonant frequency to maximise authority, which coincides with the highest tonal noise output. Since SJAs generate discrete highmomentum jets, then to induce significant effects in large scale flow they must be used in large numbers in a single array along the span of the wing.

Although there has been much research concerned with the performance optimisation of SJAs in terms of peak jet velocity output, there has been little focus on the abatement of noise generated by synthetic jets. It was observed by $\mathrm{Arik}^{2}$ that SJA jet noise can be as high as $73 \mathrm{~dB}$ when operating at a resonant frequency of $3.6 \mathrm{kHz}$ for a peak jet velocity of $90 \mathrm{~m} / \mathrm{s}$ out of a $1 \mathrm{~mm}$ orifice. Abatement techniques such as passive mufflers were shown to reduce this SJA noise down to $30 \mathrm{~dB}$, but at a cost of significantly increasing the volume of the actuator unit to a level that is unviable for practical implementation. Clarke ${ }^{3}$ suggested that a double-chamber SJA configuration can reduce the noise output based on the phenomenon of anti-phase.

The aim of the present work is to explore methods of reducing the noise generated by piezoelectrical-driven SJAs without decreasing peak jet velocity. This is to be achieved through the following objectives:

- Identify practical methods of reducing SJA noise output via configuration and/or geometric shape variations

- Design and manufacture SJA with interchangeable parts according to down-selected noise reduction methods

- Conduct a series of peak jet velocity (hot-wire anemometry) and noise (microphone recordings) experiments in order to compare conceptual SJA performance against a baseline actuator design

- Assess the electrical-to-fluidic power conversion efficiency of all actuator variations

\section{Theory}

\section{A. Noise Source}

A SJA has two main sources of noise: jet noise and structural noise. Jet noise is generated from the turbulent mixing of the flow exiting the orifice with the surrounding air. A potential core is formed just aft of the orifice exit containing laminar flow. The length of the core is typically 4 to 5 times the diameter of the orifice. The mixing of the synthetic jet with the ambient air occurs at a region around the potential core. This region is called the mixing region. Further downstream of the orifice exit, the jet spreads out at a wider angle, forming a fully-developed flow region. The vortex rings formed at the edges of the orifice increase in size and decrease in velocity as they propagate away from the SJA. The frequency of sound generated is inversely proportional to the size of the vortex rings. This means that high frequency sound is generated close to the orifice and low frequency sound derives from the fullydeveloped jet far from the orifice exit ${ }^{4}$. The continuously vibrating structural member (SJA diaphragm) creates acoustic waves inside the chamber, which bounce between the walls and finally escape through the orifice exit in to the external ambient ${ }^{5}$. 


\section{B. Noise Suppression}

\section{Double chamber SJA - dipole source}

A double-chamber SJA is essentially a double oscillating boundary 'adjacent' SJA in which there are two chambers and two orifices. The two orifice plates are located perpendicular to the oscillating diaphragm (Fig. 1). By comparison, in a single-chamber SJA the lone orifice is placed opposite or parallel to the diaphragm. The doublechamber SJA design was introduced in order to reduce noise output ${ }^{3}$. Firstly, the presence of a second chamber on the other side of the diaphragm acts as a sound barrier preventing sound waves from propagating in to the atmosphere. It is also believed that the two orifices of a double-chamber SJA act as a fluid mechanical dipole source.

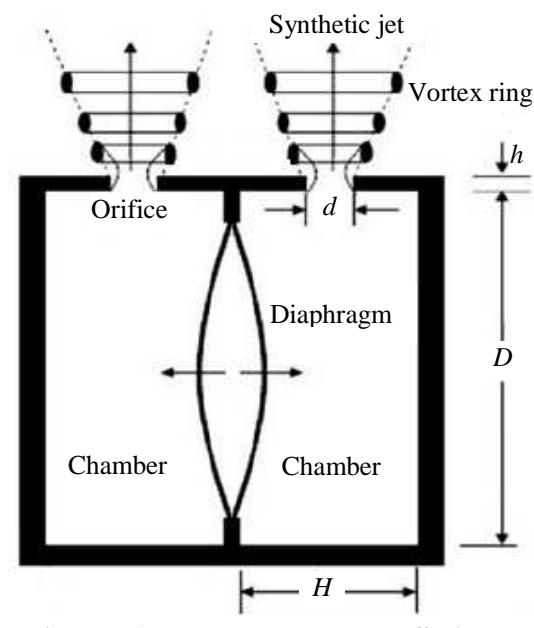

Figure 1. Double-chamber SJA

The orifice of a SJA can be characterized as a monopole source of sound (Fig. 2a). This source radiates sound in all directions; in this case, jet noise is radiated to the atmosphere. A dipole source is the close placement of two monopole sources of equal strength and exactly opposite phase ${ }^{6}$. When one source produces a net outflow, the other one produces an exactly opposite inflow. In contrast to a single monopole, the net fluid flux is zero. However, a net fluctuating force is produced because of the $180^{\circ}$ out-ofphase oscillation. In the case of a double-chamber SJA, the two orifice plates have the same dimensions (orifice diameter and depth) and are situated close to each other. As the diaphragm oscillates back and forth, flow from one orifice is exhaled while at the same time ambient air is inhaled in the other orifice. This indicates that the two orifices produce a net fluctuating source and are $180^{\circ}$ out-of-phase. Therefore, it is believed that the orifices of a double-chamber SJA are a dipole source. The propagating sound waves produced interact with each other and cancel out at $90^{\circ}$ and $270^{\circ}$ from the sound sources (Fig. 2b).

(a)

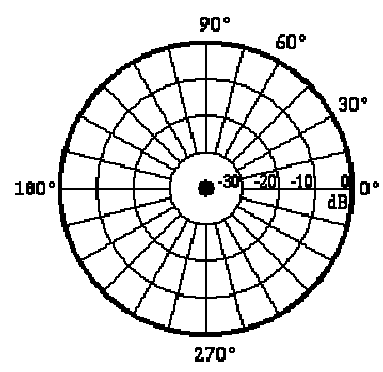

(b)

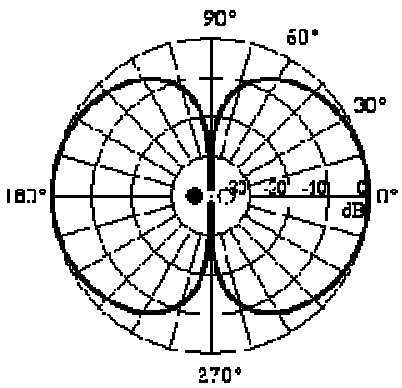

Figure 2. (a) monopole source; sound radiates in all directions, (b) dipole source; sound waves cancel out at 90 and 270 degrees from the sources

\section{Passive techniques}

There have been several nozzle design modifications devised in an effort to reduce jet exhaust noise. Research has been conducted by the aviation industry in order to specifically reduce the noise produced by aircraft jet engine nozzles. Such design modifications include chevron and corrugated nozzles. These nozzles reduce jet noise by inducing streamwise vorticity along the shear boundary layer in the jet flow. The added vorticity causes smoother mixing of the jet core with the ambient air, reducing the rapid pressure fluctuations responsible for jet noise. Enhanced mixing slightly increases the high frequency noise ranging from $7.5 \mathrm{kHz}$ to $30 \mathrm{kHz}^{7}$. However due to the breakdown of the larger scale turbulence into small scale, this mixing reduces the low frequency noise (below 7.5 $\mathrm{kHz}$ ) with result in reduction of the overall sound pressure level ${ }^{8}$.

Chevron nozzles have drawn a lot of attention recently as they are currently one of the most popular passive jet noise reduction devices. They reduce exhaust noise with minimal penalty on performance. The chevron count and penetration is a primary factor in controlling the compromise between low-frequency reduction and high-frequency SPL augmentation. It has been proven experimentally ${ }^{9}$ that a higher chevron count with a lower level of penetration 
and zero degree taper, yields the maximum noise reduction for low and medium nozzle pressure ratios. Specifically, a chevron nozzle with eight lobed chevrons reduces jet noise by $8 \mathrm{~dB}$.

For an SJA, the use of corrugated orifices (also referred to as crimped or lobed orifices) is obviously more practical due to the shape modification being in the plane of the orifice rather than out-of-plane, as is the case with chevrons. It is shown experimentally ${ }^{7}$ that an increase in the corrugation amplitude leads to noise reduction in the far field of the jet. Similar to chevron nozzles, there is a small increase of noise in the high frequency region but a decrease of noise level in the low frequency regions; typically $2.3 \mathrm{~dB}$ reduction for frequencies up to $5 \mathrm{kHz}$ compared with a non-corrugated circular orifice of the same area. The corrugated-lobed jet mixing flow has larger intensive mixing regions in the near field of the jet compared with its non-corrugated counterpart. The unique geometry of the lobes generates large scale streamwise vortices of alternating sign, thereby increasing the mixing efficiency. Consequently, the length of the potential core in the lobed jet mixing flow is $1 / 4$ to $1 / 6$ of that in the conventional circular jet flow. The performance of these nozzles depends on the configuration of the geometric parameters such as the number of lobes, their angle as well as their shape ${ }^{10}$.

\section{Sound absorption techniques}

Sound-absorbing materials convert incident sound energy into heat energy. The absorbing material has to be porous or fibrous so that the acoustic waves can oscillate within the material. Sound-absorbing material could be used inside a SJA chamber to absorb the sound waves that are produced by the diaphragm. The SJA chamber is effectively a Helmholtz resonator since air is forced in and out of it by a periodic air flow. By lining the chamber with sound absorbing material, its sound generation can be broadened over the frequency range. The sound absorption at the Helmholtz resonant frequency is slightly reduced, but at a cost of it being increased at other frequencies ${ }^{11}$. However, the lining of the chamber might affect the performance of the jet peak velocity due to energy being converted to heat.

Following a review of noise reduction techniques, it was decided to proceed with the double-chamber configuration and use of corrugated orifices to investigate their potential for noise reduction in SJAs.

\section{Design}

\section{A. SJA Geometry}

\section{Orifice}

The velocity of the exhaled synthetic jet has a great impact on SJA performance with regards to their ability to control boundary layer separation on high-lift surfaces. Peak velocities of piezoelectrically-actuated synthetic jets of the order of $80-120 \mathrm{~m} / \mathrm{s}$ have been attained ${ }^{12,13}$. As a rule of thumb, the orifice diameter should scale as a percentage of the boundary layer height in which it's embedded - typically equivalent to that of a sub boundary layer vortex generator (SBLVG) height scaling ${ }^{12,14}$. Therefore for this work, the orifice diameter, $d$, is set to $1 \mathrm{~mm}$.

The orifice height can strongly impact the peak velocity of the ensuing synthetic jet. An orifice with too large a height will tend to have a large damping effect on the airflow, resulting in a limited peak velocity. On the other hand, if the orifice neck is too short, the jet will not accelerate sufficiently to produce the desired peak velocities. The optimised height should be approximately 1.25 times the orifice diameter ${ }^{15}$. For this work, the orifice height, $h$, was constrained to $1.5 d$ (i.e. $h=1.5 \mathrm{~mm}$ ).

\section{Chamber}

The chamber diameter is constrained by the diameter of the diaphragm. The diaphragm used for this work is a polycrystalline piezoceramic (PZT-5A) disc - OBO-TE27241-16. The $27 \mathrm{~mm}$ diameter PZT diaphragm consists of a brass plate with a piezoceramic layer bonded to the alloy (total thickness of $0.45 \mathrm{~mm}$ ). PZT diaphragms have the ability to convert input electrical signals into mechanical displacements. By alternating the voltage supplied to the piezoceramic material, it contracts and expands creating vibration depending on the operating frequency range. Due to the circular shape of the disc, the vibration mode is radial and the displacement of the diaphragm is in the order of microns. The PZT disc has to be held firmly in the chamber by being clamped uniformly $2 \mathrm{~mm}$ from its perimeter. Consequently, the diameter of the chamber, $D$, is set to $25 \mathrm{~mm}$.

The peak velocity of a SJA is inversely proportional to the depth of its chamber ${ }^{15,16}$. However for a doublechamber SJA, the chamber depth is restricted by the separation distance between the two orifices. As the jets will operate in anti-phase, there is the risk that the jet being exhaled from one SJA could be inhaled during the suction 
stroke of the adjacent SJA if the orifices are positioned too close together. It was therefore decided to position the orifices $5 d$ apart $(5 \mathrm{~mm})$; this distance is also the limit of the range of optimised spacing for separation control established from experiments with arrays of SBLVGs and fluidic jets ${ }^{17-19}$. The orifice should be situated at the middle of each chamber to ensure peak velocity output. Taking into account both requirements mentioned above and the thickness of the clamped part of the diaphragm $(0.22 \mathrm{~mm})$, the depth of each chamber, $H$, in the double configuration is set to $4.78 \mathrm{~mm}$.

\section{B. Resonant Frequencies}

The theoretical resonant frequencies should be calculated in order to define the range of frequencies that the designed SJAs will be tested.

\section{Helmholtz resonance}

Helmholtz resonance occurs as a result of a dynamic exchange of kinetic energy of the fluid in the orifice with the potential (pressure) energy of the fluid in the chamber. A double-chamber SJA effectively has a pair of resonators (orifices) located close to each other producing oscillating flows which are in anti-phase. Due to the relatively small separation distance $(5 d)$ between the two resonators, the peak frequency response of both orifices is expected to increase. As previously shown ${ }^{20}$, a spanwise row of resonators increases the resonating frequency of a system in comparison to a single resonator's frequency. This effect is observed as the two orifice resonant frequencies are coupled into a new higher frequency by the fluid mechanical interaction.

The theoretical Helmholtz resonance ${ }^{21}, f_{H}$, is given by Eq. (1)

$$
f_{H}=\frac{a}{2 \pi} \sqrt{\left(\frac{d}{D}\right)^{2} \frac{1}{H h}}
$$

Where $a$ is the speed of sound. However, Eq. (1) cannot be used to determine the theoretical Helmholtz frequency of the double-chamber SJA since its use is based on a single-chamber SJA configuration. From what is known of the single-chamber SJA Helmoltz resonance $\left(f_{H}=800 \mathrm{~Hz}\right)$ and aforementioned fluid mechanical coupling between adjacent SJAs, it can be expected that the $f_{H}$ will be higher for the double-chamber configuration.

\section{Diaphragm natural resonance}

The natural or mechanical resonant frequency of the diaphragm is a key characteristic of a SJA as it often provides the greatest peak velocity output and thus tends to be more influential than the Helmholtz resonance. It is dependent on the geometric and material properties of the diaphragm. The natural frequency of a circular plate ${ }^{22}, f_{D}$, akin to a SJA diaphragm is given by Eq. (2)

$$
f_{D}=\frac{k^{2} t_{D}}{2 \pi r_{D}^{2}}\left(\sqrt{\frac{E(1-2 \xi)}{12 \rho\left(1-v^{2}\right)}}\right)
$$

Where $r_{D}$ and $t_{D}$ are the diaphragm radius and thickness respectively; $E, \rho$ and $v$ represent the diaphragm material properties (Young's modulus, density and Poisson's ratio, respectively); $\zeta$ is the damping coefficient and $k^{2}$ is a dimensionless frequency parameter, which is primarily a function of the diaphragm boundary conditions ${ }^{22}$. Table 1 summarises the diaphragm characteristics.

Table 1. SJA diaphragm charateristics

\begin{tabular}{lllllll}
\hline \hline$r_{D}(\mathrm{~mm})$ & $t_{D}(\mathrm{~mm})$ & $E(\mathrm{GPa})$ & $\rho\left(\mathrm{kg} / \mathrm{m}^{3}\right)$ & $v$ & $k^{2}$ & $\zeta$ \\
\hline 13.5 & 0.45 & 103 & 8450 & 0.33 & 4.98 & 0.06 \\
\hline \hline
\end{tabular}

The value of the natural frequency of the SJA diaphragm, $f_{D}=1960 \mathrm{~Hz}$.

\section{Modeling and Manufacture}

The SJA design is shown in Figure 3. It was necessary to design the actuator in such a way that the orifice plates are interchangeable to facilitate corrugated and non-corrugated orifice testing. 
(a)
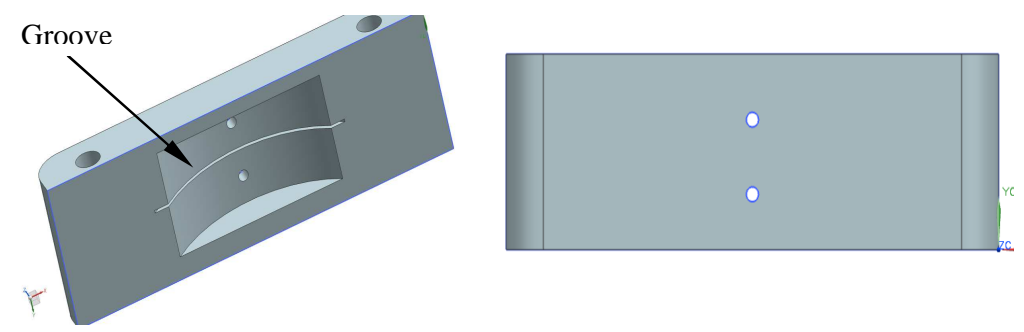

(b)
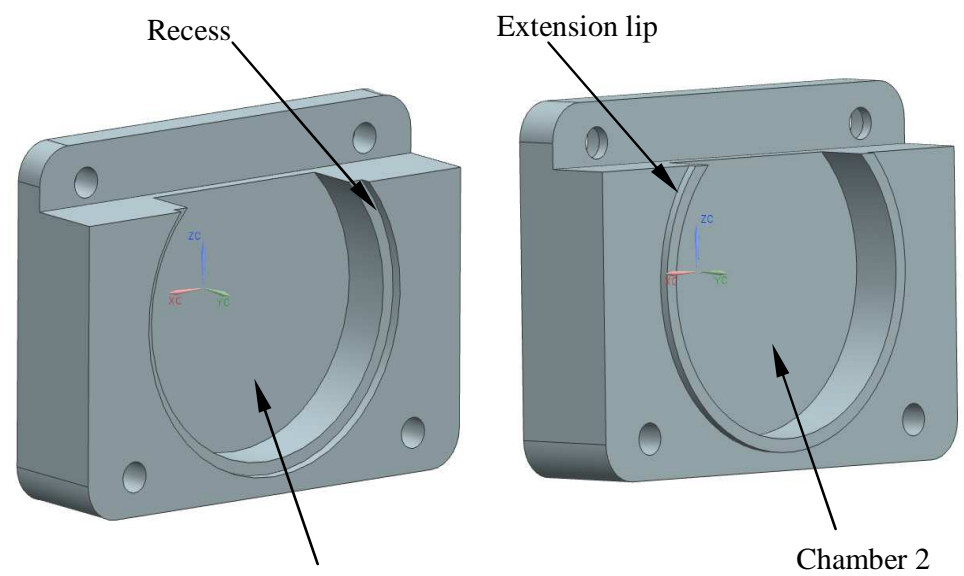

Chamber 1

Chamber 2

(c)

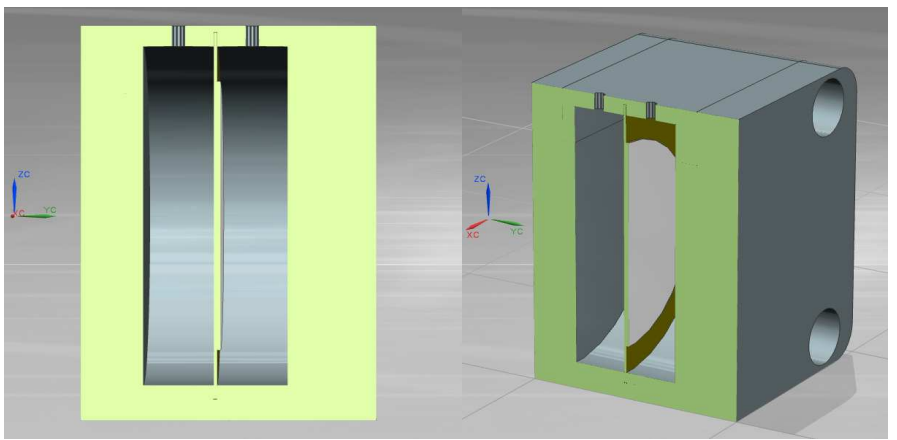

Figure 3. (a) baseline orifice plate design; (b) chamber design; (c) cross-sectional view of SJA

The maximum thickness of the orifice plate is $5 \mathrm{~mm}$ and it possesses a small groove located between the two orifices (Fig. 3a). The purpose of the groove is to locate the piezoelectric diaphragm. The double-chamber design consists of two parts: Chamber 1 and Chamber 2 (Fig. 3b). Chamber 1 has a small slot (1 mm depth), where the diaphragm will be positioned. Chamber 2 has a small extension lip $(0.78 \mathrm{~mm})$, which when assembled with Chamber 1 will provide a compressive load on the diaphragm.

A circular orifice is used as the baseline. In addition, two corrugated-lobed orifice plates were introduced for the first time in a SJA for noise reduction assessment. Kopiev et al. ${ }^{7}$ proved that a subsonic jet ensuing from an orifice with a corrugated-lobed shape has a significant decrease of jet noise at frequencies ranging from $0 \mathrm{~Hz}$ to $5 \mathrm{kHz}$. Therefore a corrugated-lobed orifice could potentially decrease the noise output of a SJA in this work, given the resonant frequencies lie within the same range. The performance of the SJA orifice will depend on the number of corrugations and the shape and amplitude of the lobes. As a minimum requirement, it is necessary to maintain the peak velocity output generated from the baseline orifice shape.

6

American Institute of Aeronautics and Astronautics 
The corrugation of the orifice was achieved by designing a dodecagon (12 sides), as shown in Fig. 4a. With 12 lines of symmetry and rotational symmetry of order 12, all sides are of equal length and all internal angles equal to $150^{\circ}$. Lobes for a given design were designed on each side of the dodecagon in a sinusoidal manner and have the same radius. In order to vary the amplitude between the two corrugated orifices, the radius of the lobes differ. The radius of the low-amplitude lobes is $0.25 \mathrm{~mm}$ (Fig. 4b) and that for the high-amplitude lobes is $0.5 \mathrm{~mm}$ (Fig. $4 \mathrm{c}$ ). The discharge rate is designed to be the same for the different corrugation amplitudes and correspond to the uncorrugated circular orifice. To achieve this, the area of the corrugated-lobed orifices should be equal to the area of the circular orifice. The 12 sides of the dodecagon have a dimension of $0.26 \mathrm{~mm}$ giving an orifice area of $7.6 \times 10^{-7}$ $\mathrm{m}^{2}$ (this is equivalent to $97 \%$ of the circular orifice area, $A=7.85 \times 10^{-7} \mathrm{~m}^{2}$ ).

(a)

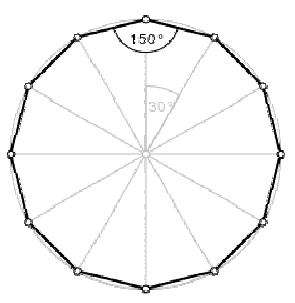

(b)

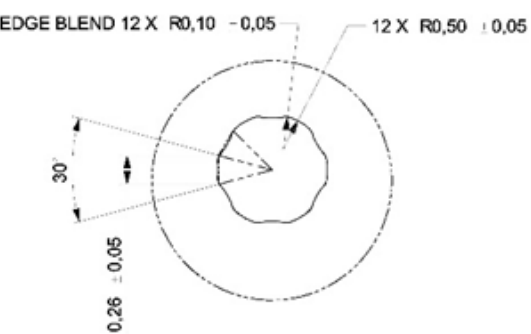

(c) EDGE BLEND $12 \times$ R0,10 $\perp 0,05-$

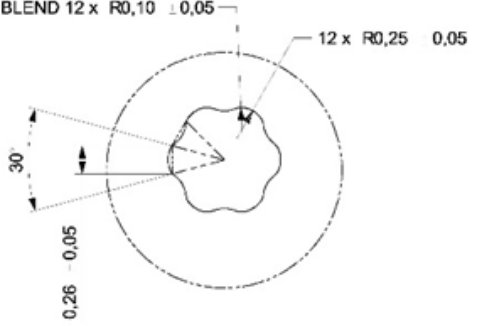

Figure 4. (a) Schematic of orifice corrugation set up; (b) low-amplitude lobed orifice; (c) high-amplitude lobed orifice

In order to manufacture parts with such small dimensions, the designs were outsourced for 3D printing. The two parts consisting of the double chamber were printed in stainless steel powder that was infused with bronze. The three interchangeable orifice plates were printed using a high grade stainless steel (316L) which, due to its tight tolerances, would potentially deliver better results on the level of detail in the corrugation. All parts were created by binding together layers of stainless steel powder by using powerful heaters. After 3D printing was completed, the parts were placed in a curing oven for sintering.

\section{Experimental Approach}

\section{A. Jet Velocity Measurement}

The SJA drive components are shown in Fig. 5. A Trek PZD350A high-voltage piezo driver/amplifier with an output voltage range of 0 to $\pm 350 \mathrm{~V}$ DC or peak $\mathrm{AC}$ was used to provide power to the piezoelectric diaphragm. The voltage signal sent to the PZT disc from the amplifier was controlled by a TTI TG215 $2 \mathrm{MHz}$ function generator. Variation in voltage and frequency results in different modes of disc oscillations hence affecting the velocity output. A fixed sinusoidal voltage waveform was used. Two digital bench multimeters (Model UT801) were connected to the piezo amplifier to provide readings of voltage and current input. Each SJA was tested with peak-to-peak voltage input, $V_{p p}=100 \mathrm{~V}$ at excitatation frequencies $800 \mathrm{~Hz}$ to $3 \mathrm{kHz}$ in increments of $100 \mathrm{~Hz}$.

Measurement readings of peak velocity were acquired from a Dantec Dynamic 55P11 single hot wire anemometer sensor probe, which interfaces with a CTA module frame controller. The controller includes a BNC input used for monitoring the temperature of the probe, calibration and data acquisition. Data is acquired through Streamware Pro System software and subsequently exported to Excel for detailed analysis. In order to ensure that the peak velocity output of the SJA is captured, the probe positioning is of high importance. The hot-wire probe was positioned $1 \mathrm{~mm}$ from the orifice exit using a vertical micrometer. Since the hot-wire has a length of $1.25 \mathrm{~mm}$, it was centred with the wire covering the entire orifice width. 


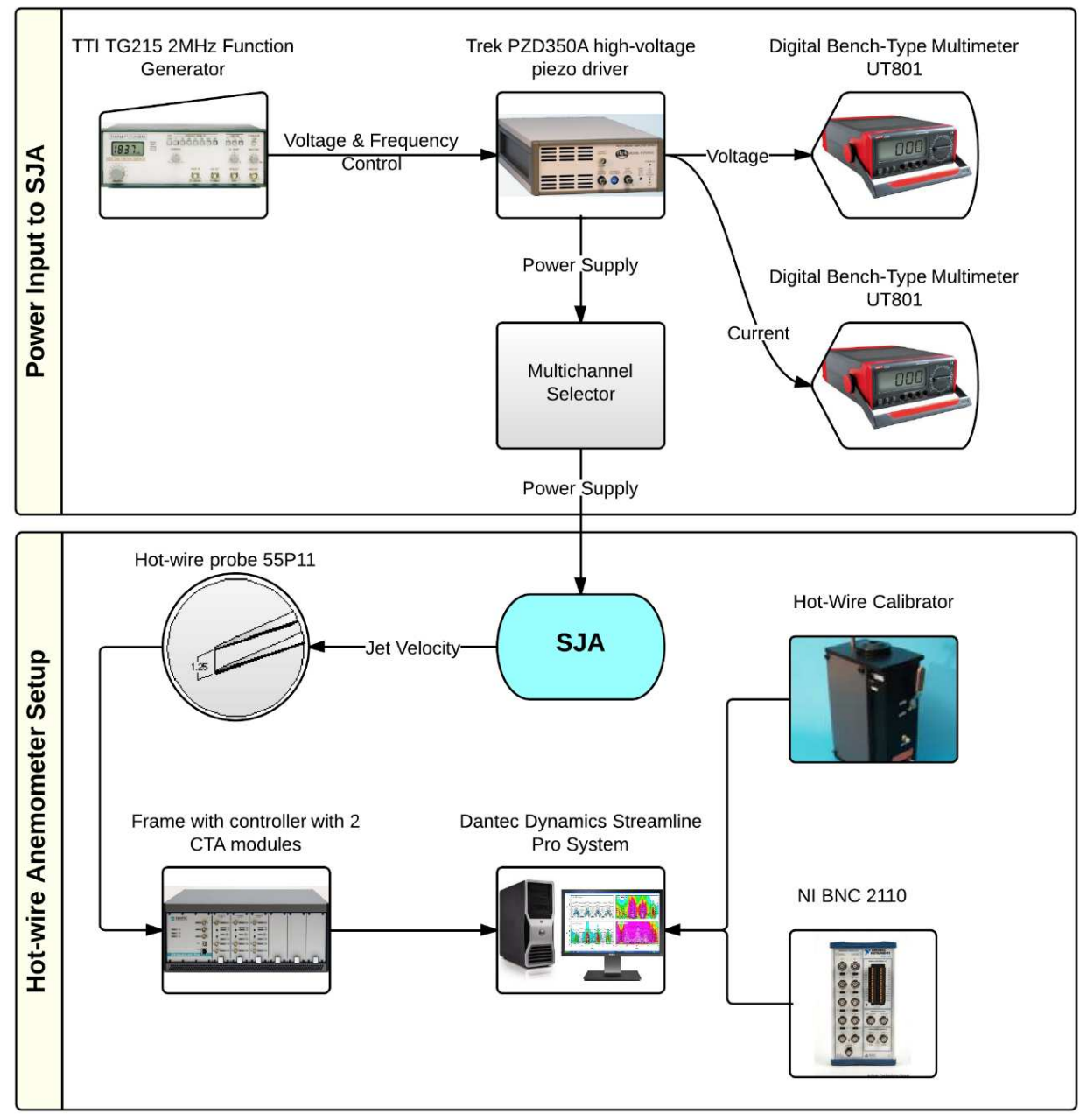

Figure 5. SJA jet velocity measurement setup

\section{B. Power Conversion Efficiency Measurement}

The power supplied to a SJA can be considered as electric energy input, $\dot{E}$. Some of this energy is stored as electrical potential energy because of the electric capacitance of the PZT diaphragm, which is denoted as $\dot{C}$. The remaining energy is converted to mechanical energy along with energy loss. The mechanical energy of the actuator can be considered as the vibration of the piezoelectric diaphragm plus the synthetic jet exiting the orifice. For this project, it is necessary to determine the electric-to-fluidic power conversion efficiency of the SJA, thus the mechanical energy of the diaphragm due to vibration is not considered. It is assumed that the static pressure and specific volume of air at the orifice are the same as the ambient. Therefore, the energy of airflow exiting the orifice is equal to its kinetic energy, $\dot{F}$. The energy loss of the SJA is incurred due to the deflection of the diaphragm as well as some energy loss in airflow prior to exiting the orifice. The total energy loss is represented by $\dot{L}$. Figure 6 shows the rate of input and output energy conversion of a single and double chamber SJA. The square area is the control volume of the system (SJA).

For double-chamber SJAs (Fig. 6b), the flow power will be higher due to the ejection of two synthetic jets with the same amount of power input. The efficiency of energy conversion for synthetic jets is the ratio of the output and input energy given by

$$
\eta=\frac{\dot{F}}{\dot{E}}
$$


(a)

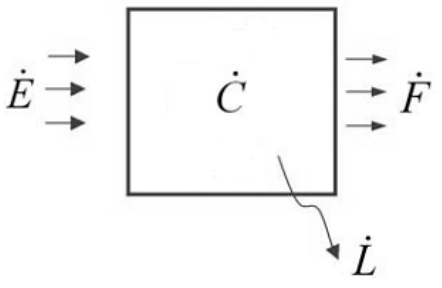

(b)

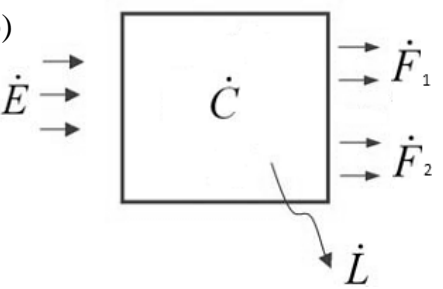

Figure 6. Energy conversion comparison between (a) single- and (b) double-chamber SJA

To calculate the efficiency, it is necessary to determine the flow power. The airflow power rate for a single chamber $\mathrm{SJA}^{23}$ can be derived by

$$
\dot{F}=\frac{1}{2} \rho A u^{3}
$$

Where $A$ is the orifice area of the SJA, $\rho$ is the density of air and $u$ is the synthetic jet velocity.

However, a double-chamber configuration has two synthetic jets. Therefore, the airflow power rate for both synthetic jets is determined by

$$
\dot{F}=\dot{F}_{1}+\dot{F}_{2}
$$

The power input applied to the piezoelectric diaphragm depends on the voltage amplitude. Due to the electrical capacitance of the diaphragm, there is a phase angle difference between the voltage and current. Because of that, the current signal has a time delay that varies over a range of frequencies. The phase angle is given by

$$
\phi=2 \pi f t
$$

The instantaneous true power input is calculated as

$$
\dot{E}=V_{\text {peak }} I_{\text {peak }} \sin (2 \pi f t) \sin (2 \pi f t+\phi)
$$

Where $V_{\text {peak }}$ and $I_{\text {peak }}$ is the peak voltage and current respectively, $f$ is the excitation frequency and $t$ is the instantaneous time.

Since the experimental velocity measurements were compared at peak velocities, the flow and electrical power are calculated at peak values. It is necessary to obtain an expression for the peak electrical power. The derivation is shown below:

From trigonometry:

$$
\dot{E}=V_{\text {peak }} I_{\text {peak }} \sin (2 \pi f t) \sin (2 \pi f t+\phi)
$$

$$
V_{\text {peak }} I_{\text {peak }} \sin (2 \pi f t) \sin (2 \pi f t+\phi)=V_{\text {peak }} I_{\text {peak }}\left(\frac{1}{2} \cos (\phi)-\cos (4 \pi f t+\phi)\right)
$$

Equation 7 is a function of time and will give the maximum peak true power when the term $\cos (4 \pi f t+\phi)=-1$. The rest of the terms remain constant at a given frequency. Therefore

$$
E_{p e a k}=\frac{V_{p e a k} I_{p e a k}}{2} \cos \phi+\frac{V_{p e a k} I_{p e a k}}{2}
$$

where $E_{\text {peak }}$ is the peak power input and $\cos \phi$ is the power factor, which is equivalent to the phase angle $(\phi=2 \pi f t)$.

During jet velocity measurements, voltage and current data were additionally acquired in order to calculate the peak electrical-to-fluidic power conversion efficiency. The power input was calculated based on Eq. 8; the phase difference between the voltage and current signals was captured using a digital oscilloscope, PicoScope.

9

American Institute of Aeronautics and Astronautics 


\section{Jet Noise Measurement}

The SJA jet noise measurement components are shown in Fig. 7. The microphone set consists of a GRAS 1/4" Type 40BH precision microphone and a 1/4" preamplifier Type 26TC with integrated $3 \mathrm{~m}$ cable with a 7-pin LEMO connector. The $40 \mathrm{BH}$ precision microphone is usually used for high level acoustic measurements. It's very low sensitivity and wide frequency response make it ideal for the SJA noise experiments where it is expected to acquire measurements of the order of 60-70 dB at the resonant frequencies. A GRAS Power Module Type 12AK is used in order to supply power to the microphone set via a 7-pin LEMO input socket. The power module has an output socket which is connected through a BNC cable to National Instrument BNC 2100 for data logging. The filter and gain settings were linear and $+50 \mathrm{~dB}$, respectively.

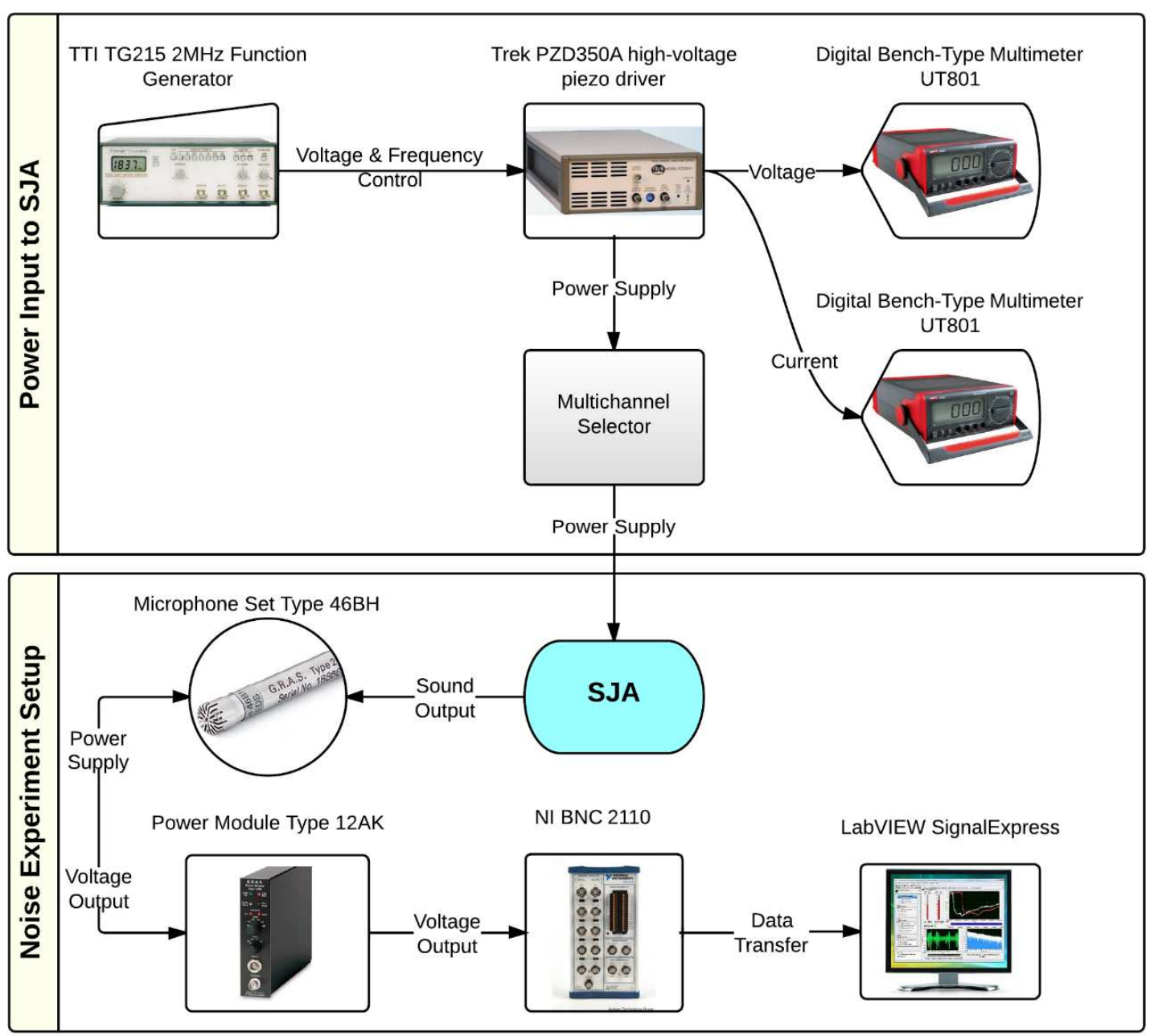

Figure 7. SJA jet noise measurement setup

The noise experiments were conducted in a purposely built test rig, which is essentially a sound proof Perspex box that simulates an anechoic chamber (Fig. 8). Pyramid-shaped acoustic panels made from polyurethane foam were installed on the inside walls of the test rig to limit echoes and sound wave reflections and to act as a sound absorber to minimize external noise affecting the test measurements. A removable base was necessary for ease of placement of the SJA inside the box. The SJA was firmly secured on a vice which is mounted on the centre of the base plate. Acoustic foam was also placed between the SJA and vice to eliminate sounds due to vibrations transferred from the actuator to the vice. The microphone was placed inside the box through a 1/2" aperture located at the centre of the ceiling and at a distance of $10 \mathrm{~cm}$ from the SJA orifice plate. Test parameters (SJA input voltage and excitation frequency range) for the jet velocity measurements were replicated for the jet noise measurements. 

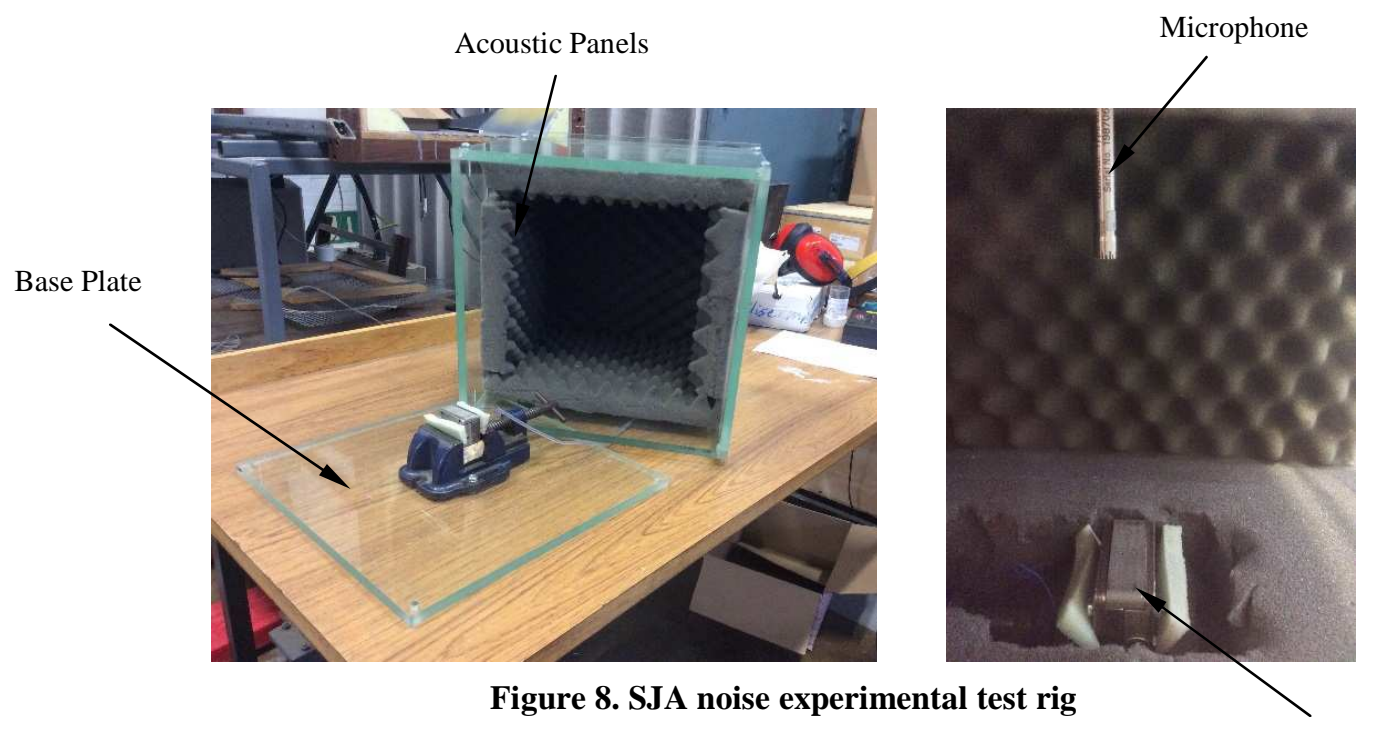

Figure 8. SJA noise experimental test rig

The microphone's voltage output values were captured in LabVIEW. The voltage signal wave is sinusoidal, therefore in order to determine the peak sound pressure level at a given frequency, it was necessary to extract the peak voltage amplitude for positive and negative values. The peak-to-peak voltage was calculated and was used to convert the voltage output into sound pressure level (SPL) of the SJA in decibels as follows

$$
S P L=20 \log _{10}\left(\frac{V_{p p}}{V_{r e f}}\right)-\text { mic sensitivity }+ \text { calibration input signal }- \text { amp gain }
$$

where $V_{p p}$ is the peak-to-peak voltage output of the microphone and $V_{r e f}$ is the transform factor of the microphone $(\mathrm{mV} / \mathrm{Pa})$. Microphone sensitivity and calibration signal are $-66.13 \mathrm{~dB}$ re. $1 \mathrm{~V} / \mathrm{Pa}$ and $114 \mathrm{~dB}$, respectively. The absolute SPL values for each orifice plate were filtered by applying an A-weighted factor in an effort to account for the relative loudness perceived by the human ear. The jet noise results are therefore presented in units of dBA.

D. Experimental Uncertainty

1. Manufacturing discrepancies

(a)

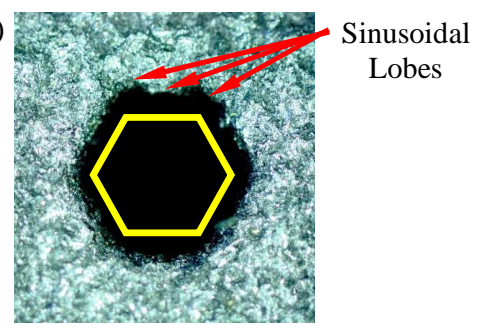

(b)

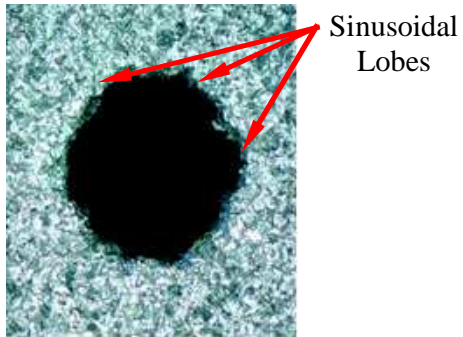

A number of manufacturing discrepancies were discovered with the orifice plates. The diameter of the circular orifices was optically measured using a TESA-VISIO 200 optical measurement machine. The actual diameters of the two circular orifices were measured as $0.885 \mathrm{~mm}$, which is $0.115 \mathrm{~mm}$ less than the design specifications of $1 \mathrm{~mm}$. Due to that relatively minor discrepancy, it is expected that the peak output velocity and noise will be slightly affected. Moreover, Helmholtz resonant frequency will decrease to some extent relative to the theoretical value (Eq. 1). The high and low corrugated plates were also placed under the digital microscope for examination of their shape (Fig. 9). It was observed that the high amplitude corrugated-lobed orifice has sinusoidal lobes, but they are not always distinct around the perimeter; similarly so for the low amplitude corrugated lobes. On the other hand, the amplitude of the lobes between the two orifice types is different and the corrugation is quite distinct as expected. The fact that the sinusoidal lobes are not very distinct will affect the enhancement mixing of the turbulent synthetic jet with the ambient air and thus the results in noise reduction may not be as large as those discussed in the literature.

Figure 9. 20x magnification of SJA orifice: (a) high amplitude corrugation; (b) low amplitude corrugation 


\section{Measurement discrepencies}

Despite the fact that the calibration of the hot-wire probe is performed with a dedicated calibrator, it is a key source of uncertainty. The calibration errors are stochastic with normal distribution and usually the calibration error is approximately $\pm 1 \%$. Depending on the positioning of the probe during the experimental setup, positioning errors may vary across the tests. The uncertainty is described as stochastic with a square distribution and can be expressed as $u\left(u_{\text {position }}\right)=1 / \sqrt{3}(1-\cos \theta)$. On average, the probe positioning error is approximately in the order of $\Delta \theta=1^{\circ}$.

The double-chamber SJA consists of 3 interchangeable orifice plates with different designs. Ideally, the velocity measurements would be captured from both orifices of a plate simultaneously from 2 different probes of the same type. In this case, if room conditions such as temperature and pressure changed, it would equally affect the results of both orifices. However, due to the limited number of probes, the synthetic jet velocity of each orifice had to be measured separately. This method could potentially induce uncertainties in the results if ambient conditions were changed between the two experiments. In an effort to minimise these kind of uncertainties, the testing of both orifices of a single plate were performed in sequence within a very short period of time to ensure similar ambient conditions in terms of temperature and barometric pressure.

The uncertainty of the microphone measurement is $\pm 0.06 \mathrm{~dB}$. Sensitivity was measured with an acoustic input signal of $114 \mathrm{~dB}$ at $250 \mathrm{~Hz}$, generated by a GRAS Pistonphone Type 42AP, and is traceable to National Physics Laboratory, UK. All tests runs were performed on the same day to ensure that the environmental conditions such as temperature, humidity, ambient noise and barometric pressure are uniform across all experiments. Furthermore, all variables such as the positioning of the actuator on the test rig and PZT disc clamping conditions remained constant where possible throughout the entire test procedure.

\section{Results}

\section{A. SJA Jet Velocity Output}

The raw data acquired from the hot-wire experiments were processed in Excel to find the SJA peak velocity response to excitation frequency. The peak velocity was identified within the data for each frequency sweep. Streams of 256 samples were collected at a sampling rate of $40 \mathrm{kHz}$ for each frequency setting, which were converted into velocity according to the hot-wire calibration curve and subsequently plotted as $2 \mathrm{D}$ graphs.

\section{Single- and double-chamber comparisons}

In order to assess whether there is fluid mechanic interaction between the oscillating flows at the orifices of the double-chamber SJA, Orifice 2 was blocked with tape to simulate a single-chamber SJA. Velocity measurements were taken for Orifice 1 of the circular and low corrugated orifces at $100 \mathrm{~V}_{p p}$ and the results are shown in Fig 10a and $10 \mathrm{~b}$ respectively. It is observed that Orifice 1 behaves in a very similar manner in both double and single chamber configurations. The output peak velocity variation across all frequency responses is less than $5 \mathrm{~m} / \mathrm{s}$ between the two arrangements. The mechanical resonance of the diaphragm is equally noticeable at $1.7 \mathrm{kHz}$ with a peak jet velocity of approximately $37 \mathrm{~m} / \mathrm{s}$.

Similarly for the low amplitude corrugated orifice, single and double chamber SJAs have the same behaviour up to $2 \mathrm{kHz}$. At higher frequencies, the single chamber configuration has much higher velocities (up to $20 \mathrm{~m} / \mathrm{s}$ ). However, it is not believed that this is due to interference of the flow between the 2 orifices. Experimental error could have occurred due to inaccurate positioning of the hotwire probe that would have lowered the reading values at that instance. From Fig. 10, it can therefore be seen that single and double chamber SJAs have similar magnitudes of peak jet velocities for different orifice designs. In can also be reasonably concluded that, in the double-chamber SJA configuration, the two orifices at a distance of $5 d$ apart do not have fluid interaction with each other that causes the jet velocity to be diminished.

\section{Orifice shape comparisions}

It can be seen from Fig. 11a that the velocity output of the two orifices of the circular plate have a similar trend. A maximum peak jet velocity of $38 \mathrm{~m} / \mathrm{s}$ and $34 \mathrm{~m} / \mathrm{s}$ was obtained at $1.7 \mathrm{kHz}$ for Orifice 1 and Orifice 2 respectively. The frequency response is dominated by a single peak, suggesting strong coupling between the Helmholtz resonance and the natural frequency of the diaphragm. The peak at $1.7 \mathrm{kHz}$ correlates strongly with the theoretical value of diaphragm resonance as this tends to be more influential than the Helmholtz resonance. 
(a)

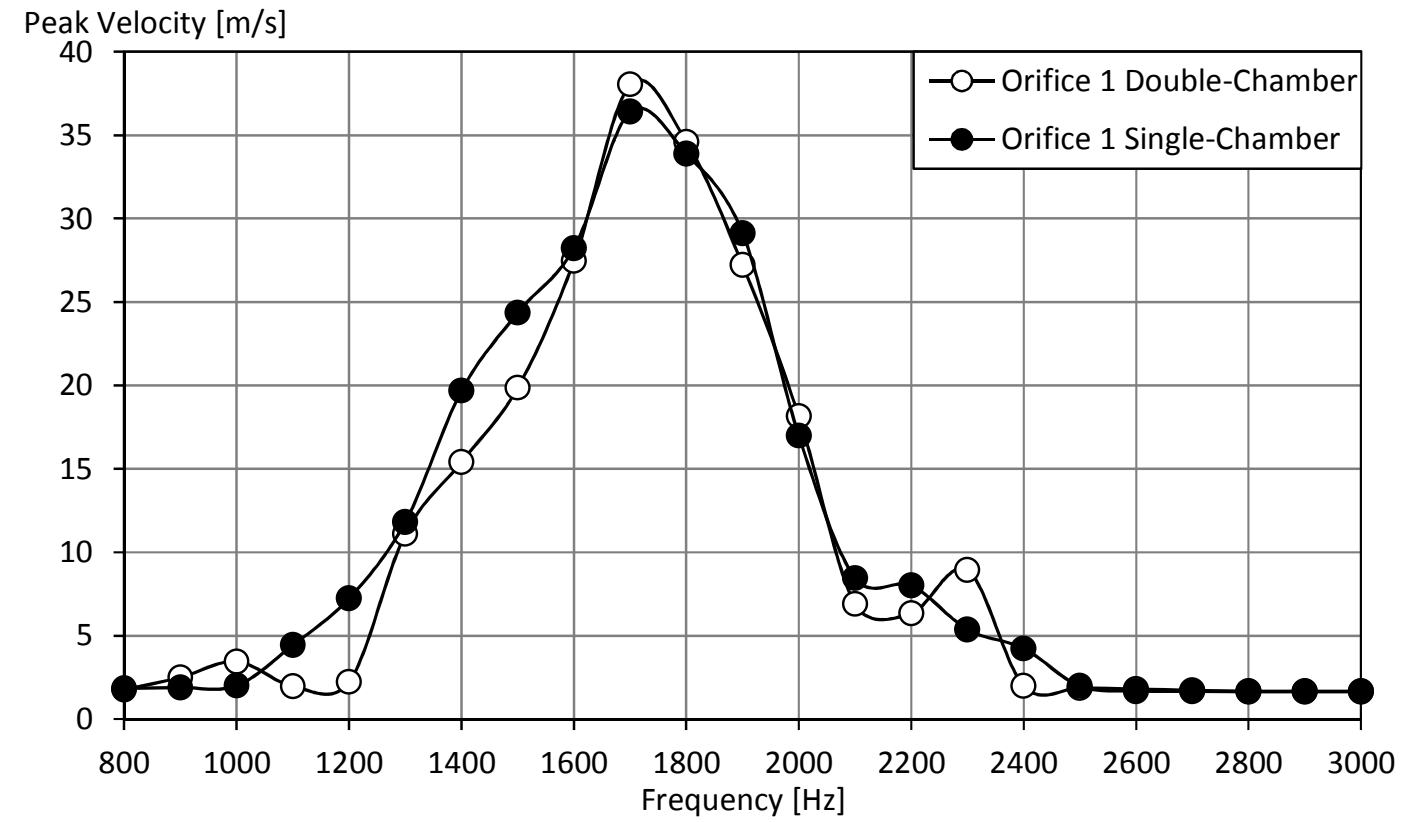

(b) Peak Velocity $[\mathrm{m} / \mathrm{s}]$

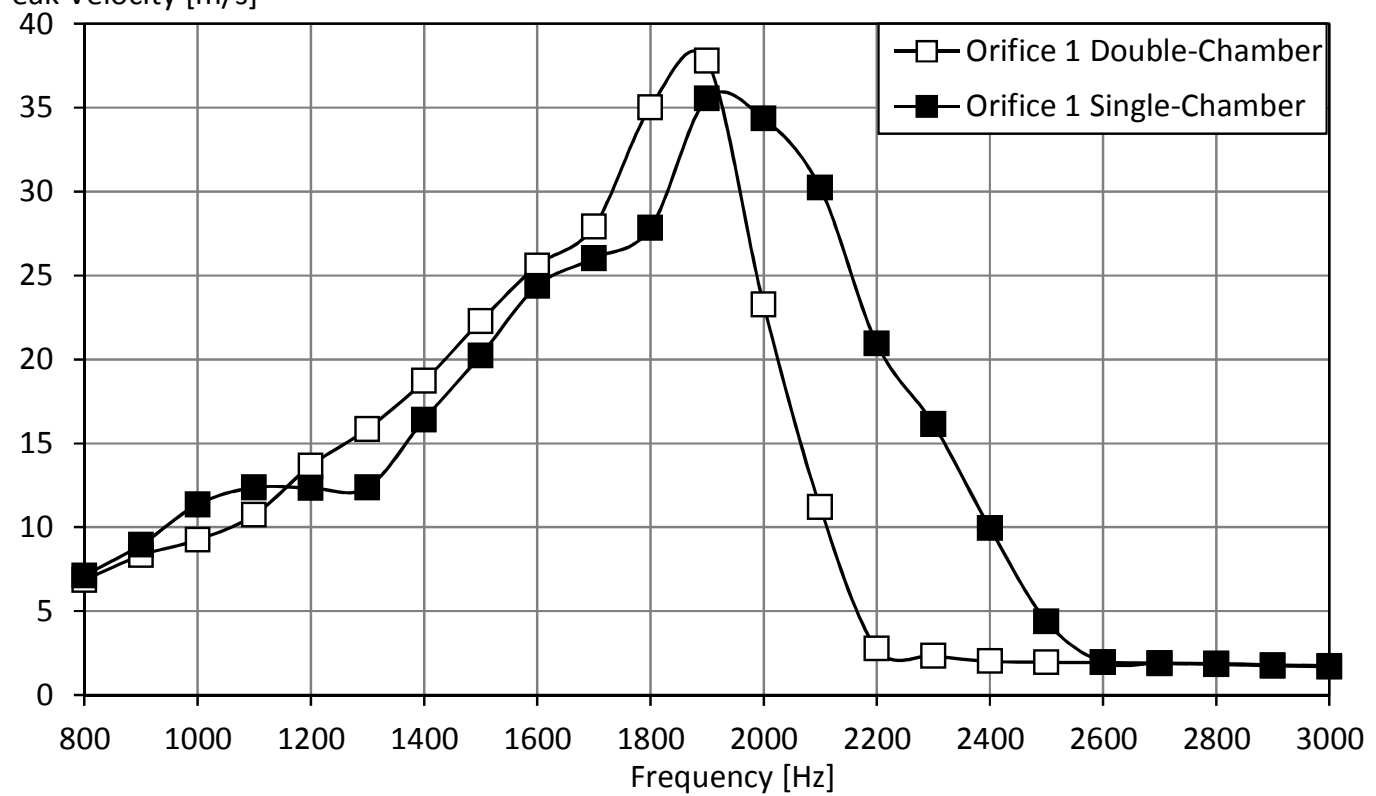

Figure 10. Comparison between single- and double-chamber SJA configurations: (a) circular orifice; (b) lowamplitude corrugated lobed orifice

Figure $11 \mathrm{~b}$ shows the peak velocities of the double-chamber SJA with low-amplitude corrugated lobed orifice. A maximum peak velocity of $38 \mathrm{~m} / \mathrm{s}$ and $32 \mathrm{~m} / \mathrm{s}$ for Orifice 1 and 2 respectively is observed at $1.9 \mathrm{kHz}$. The magnitude of the velocity response is very similar to that observed for the circular orifices. Figure 11c presents the high amplitude corrugated-lobed SJA data. The Helmholtz and natural frequency are clearly coupled and occur at 1.5 $\mathrm{kHz}$ and $1.7 \mathrm{kHz}$ respectively as seen by the two distinctive peaks. The maximum peak velocity recorded was 35 $\mathrm{m} / \mathrm{s}$ for Orifice 1 and $38 \mathrm{~m} / \mathrm{s}$ for Orifice 2 at $1.7 \mathrm{kHz}$. 
(a)

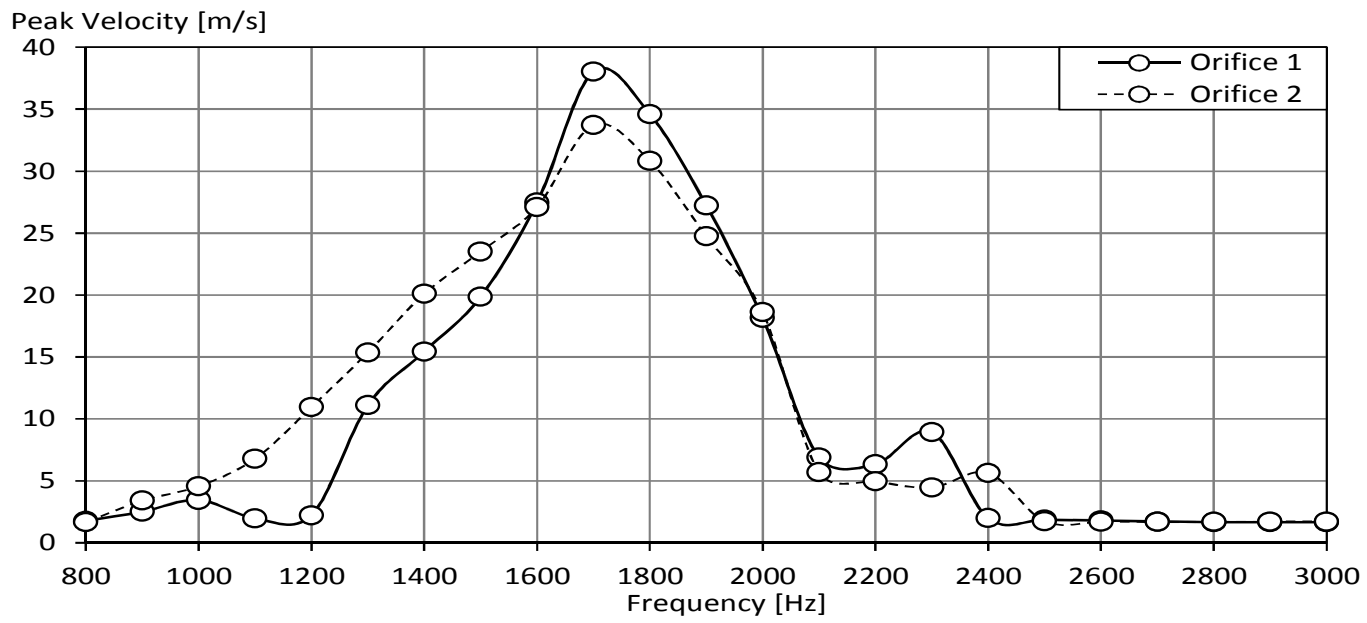

(b)

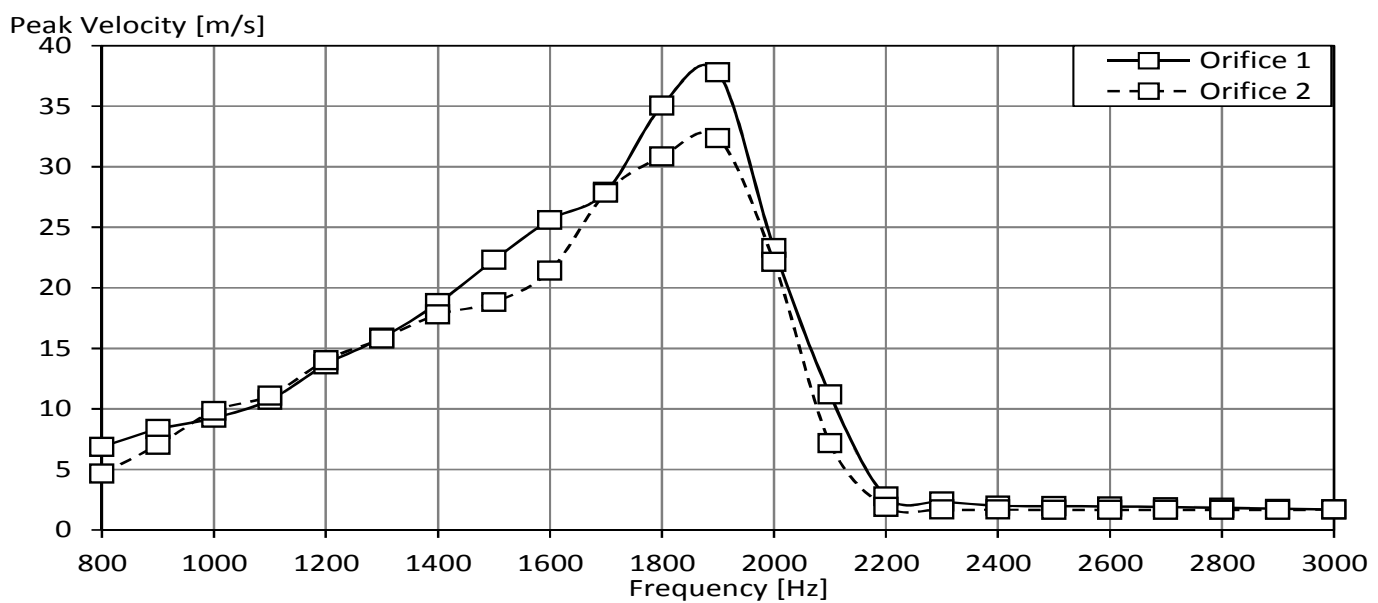

(c)

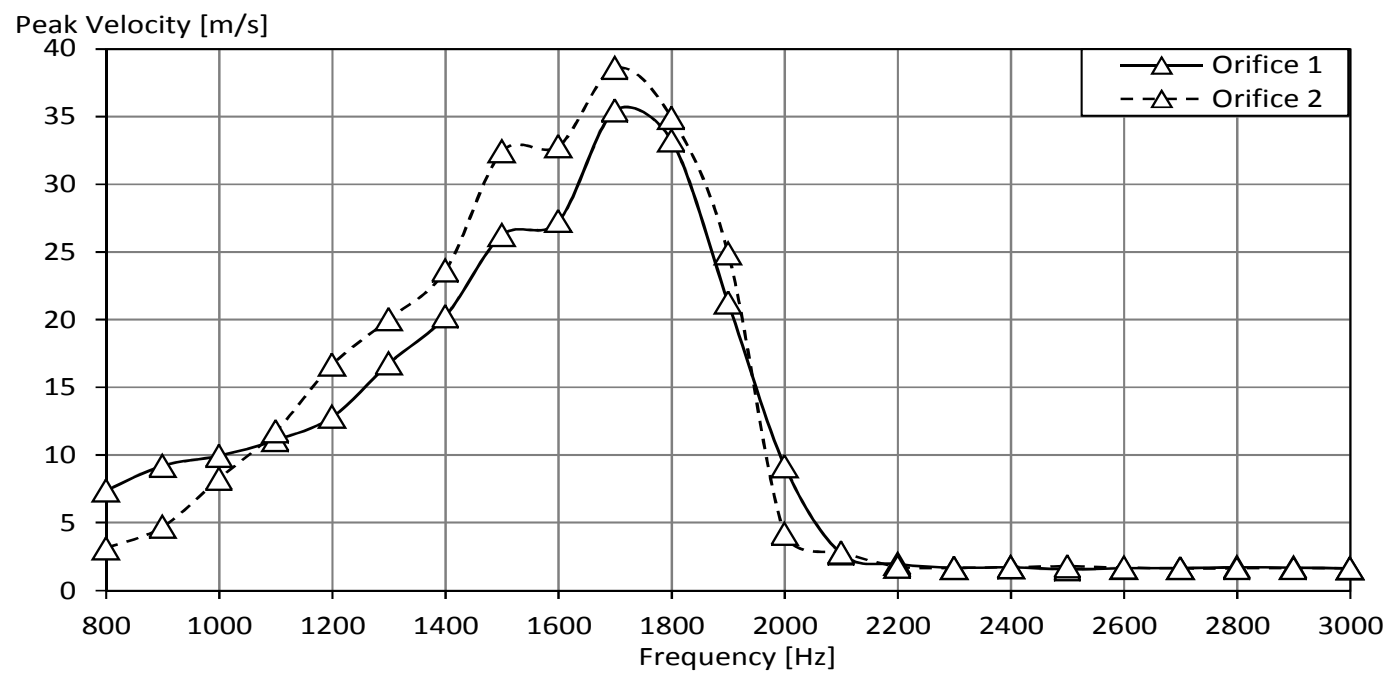

Figure 11. Comparison between orifice shapes in the double-chamber SJA configurations: (a) circular orifice; (b) low-amplitude corrugated lobed orifice; (c) high-amplitude corrugated lobed orifice

American Institute of Aeronautics and Astronautics 
Figure 12 summarise the Orifice 1 peak velocities of each orifice shape plate as a function of excitation frequency. It is observed that the circular orifice has the maximum peak velocity output $(38.04 \mathrm{~m} / \mathrm{s})$ in comparison to the low and high corrugated-lobed orifices. This was expected because most of the turbulent kinetic energy in the corrugated or lobed-shaped mixing is dissipated in the intensified mixing process, thus kinetic energy and jet velocity begin to decrease. The maximum peak velocity of the low amplitude corrugated-lobe plate was $37.79 \mathrm{~m} / \mathrm{s}$ while for the high corrugation it was $35.46 \mathrm{~m} / \mathrm{s}$. Importantly, these are still very close to uncorrugated case. It was also observed that the mechanical and Helmholtz resonance for the circular and high corrugated orifice occurred at $1.7 \mathrm{kHz}$ and $1.5 \mathrm{kHz}$ respectively. However, the natural frequency of the PZT disc for the low corrugated orifice configuration was seen at $1.9 \mathrm{kHz}$. It is believed that the difference in this resonance was due to slightly unequal clamping conditions across the 3 plates.

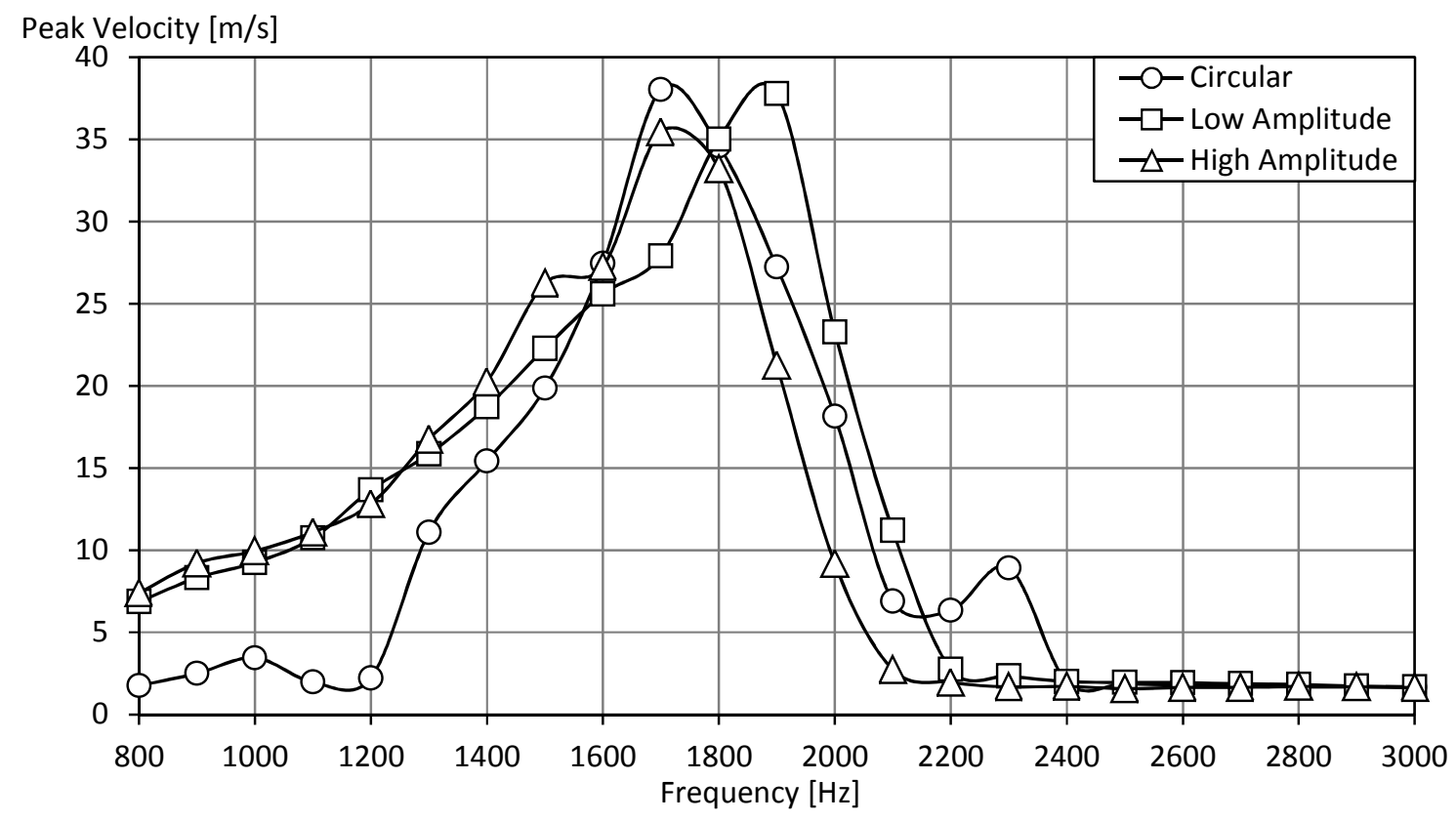

Figure 12. Peak jet velocity - comparison between orifice shapes in the double-chamber SJA

\section{B. SJA Power Conversion Efficiency}

Figure 13a shows the electrical-to-fluidic power conversion efficiency of the circular orifice SJA configuration at 100 and $200 \mathrm{~V}_{p p}$. Efficiency varies from 0 to $\sim 15 \%$ at $100 \mathrm{~V}_{p p}$ and from 0 to $\sim 8 \%$ at $200 \mathrm{~V}_{p p}$. This indicates that at $200 \mathrm{~V}_{p p}$ the jets output higher flow power, but as a consequence of consuming significantly more electric power. It can also be seen that the efficiency varies with frequency. The SJA is most efficient when it is operated around 1.7 $\mathrm{kHz}$ for both voltage amplitudes. This frequency coincides with the mechanical resonance of the diaphragm where the maximum peak velocity is produced.

Figure $13 \mathrm{~b}$ presents the efficiency of the low corrugated orifice at 100 and $200 \mathrm{~V}_{p p}$. Similar to the circular orifice, the efficiency at both driving voltages follows the same trend. The maximum efficiency is observed at 1.9 $\mathrm{kHz}$ where the natural frequency of the diaphragm occurred. The efficiency at $100 \mathrm{~V}_{p p}$ is again higher than at 200 $\mathrm{V}_{p p}$, although above $2.4 \mathrm{kHz}$ the difference is minimal. This is due to the fact that the electrical power input is solely converted into mechanical vibration of the diaphragm and heat loss. The velocity output at those high frequencies is zero and therefore no fluidic power is generated.

A peak efficiency of around 12.5 per cent is obtained at $100 \mathrm{~V}_{p p}$ and excitation frequency of $1.7 \mathrm{kHz}$ for the high corrugated orifice (Fig. 13c), corresponding to a jet velocity of $38 \mathrm{~m} / \mathrm{s}$. When the input voltage is increased to 200 $\mathrm{V}_{p p}$, the peak efficiency is reduced by $2 \%$. At $100 \mathrm{~V}_{p p}$, there are 2 distinct efficiency peaks that occur close to the Helmholtz and natural resonant frequencies.

Overall, peak efficiencies are comparable for all orifice designs at a given input voltage. 
(a) Efficiency [\%]

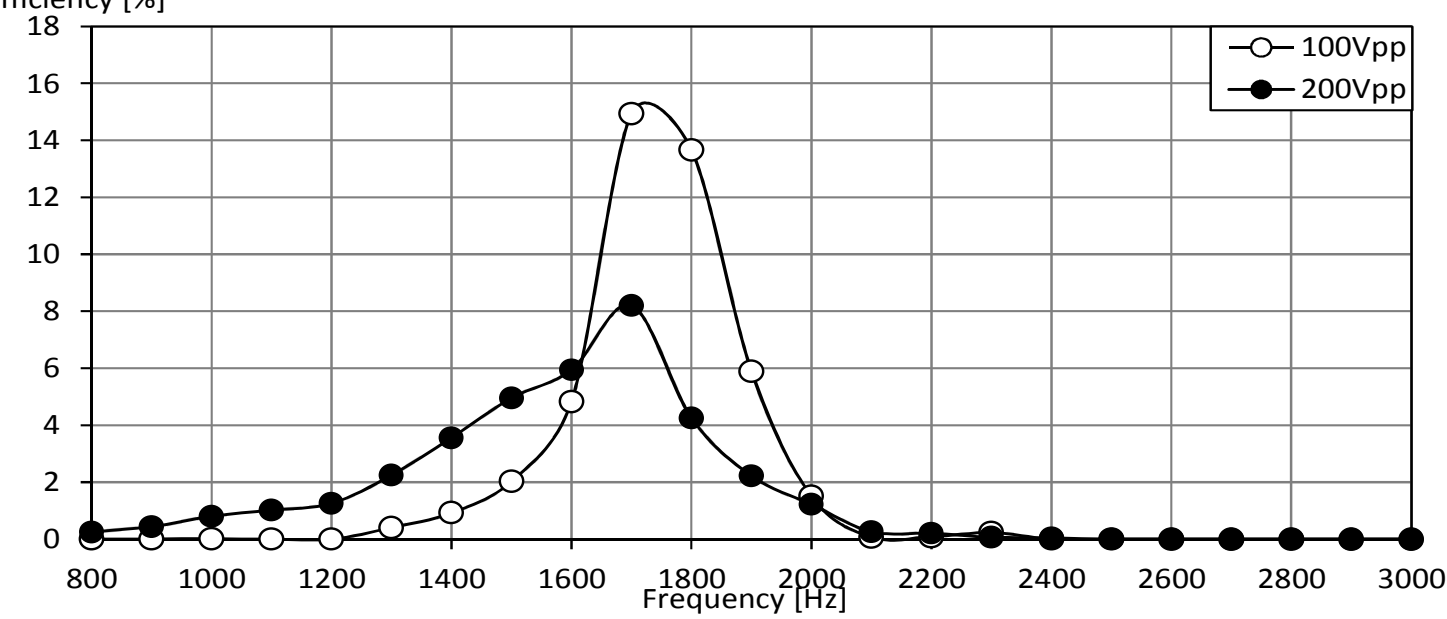

(b) Efficiency [\%]

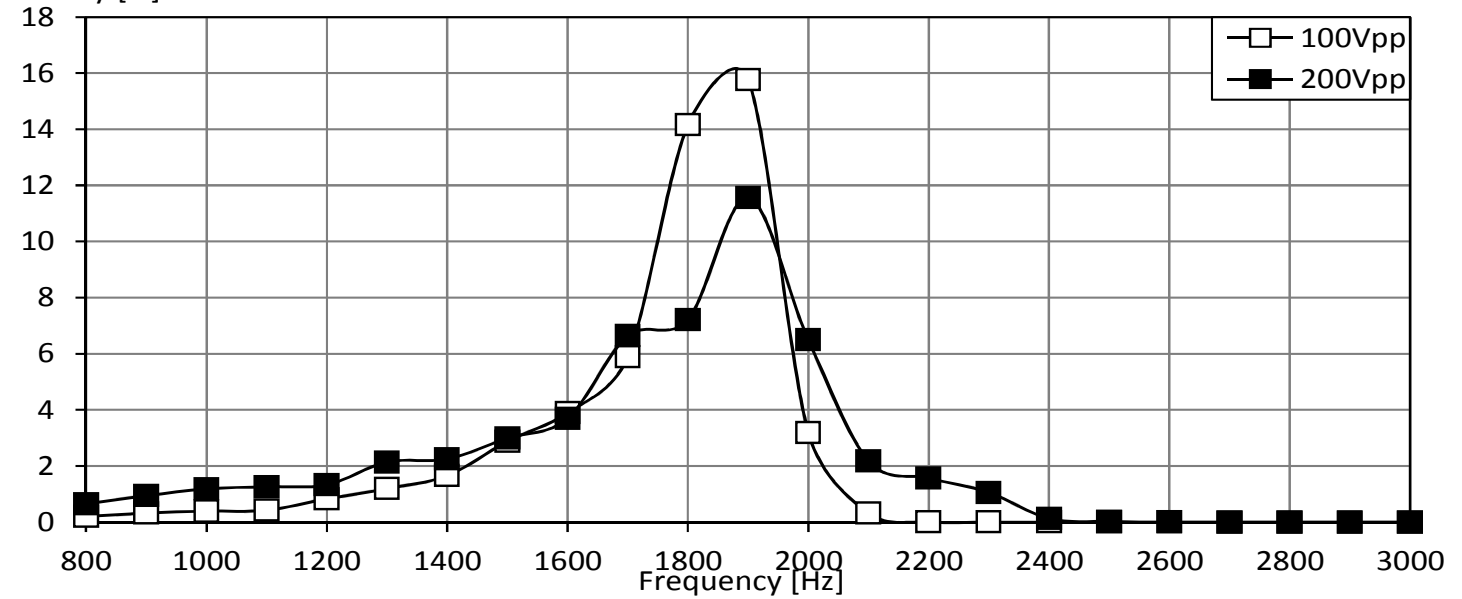

(c)Efficiency [\%]

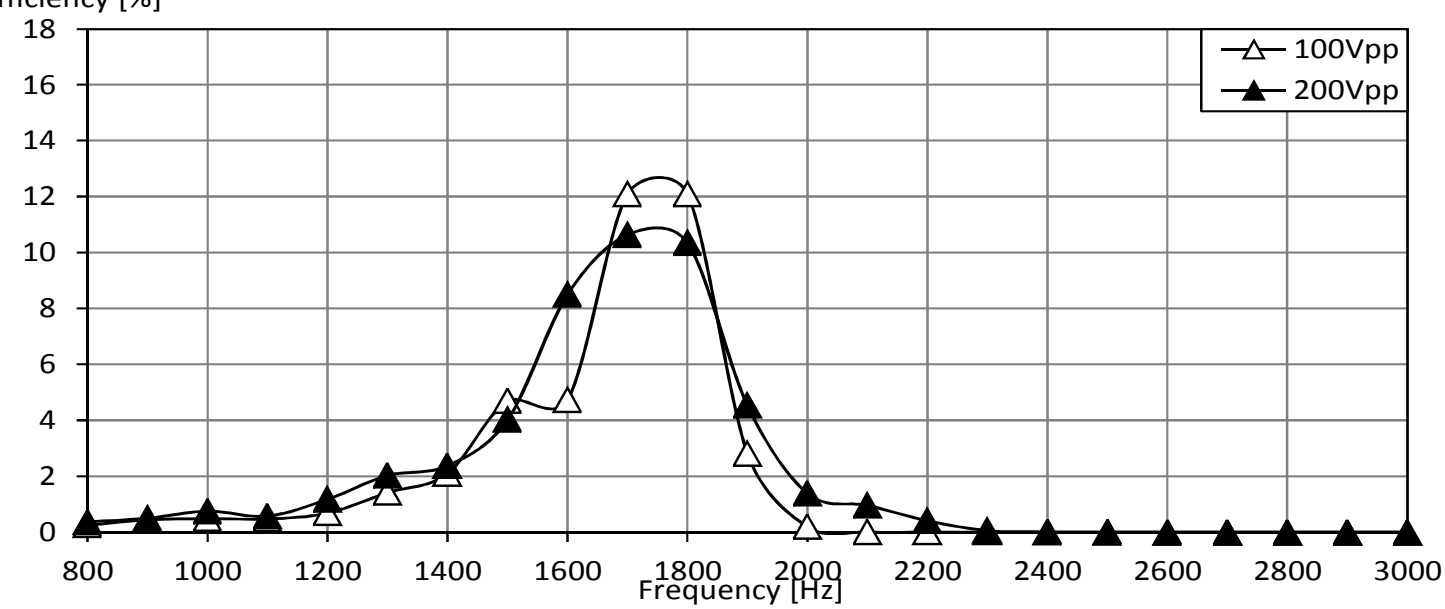

Figure 13. Power conversion efficiency - comparison between orifice shapes in the double-chamber SJA: (a) circular orifice; (b) low-amplitude corrugated lobed orifice; (c) high-amplitude corrugated lobed orifice 


\section{SJA Jet Noise Output}

The SJA was placed in the noise measurement test rig and was recorded while operating at a range of frequencies. In order to measure the extent of the anti-phase noise reduction, the SJA was tested with 3 different orifice designs as a double-chamber actuator. Then all tests were repeated with one of the orifices blocked with tape to simulate a single-chamber actuator.

\section{Single- and double-chamber comparisons}

Figures $14 \mathrm{a}, 14 \mathrm{~b}$ and $14 \mathrm{c}$ present the noise output of the SJA for single and double chamber configuration of the circular, low and high corrugated-lobed plates respectively. It can be observed in all three cases that the singlechamber SJA has a louder noise output than the double-chamber configuration. This phenomenon which is similarly noticed in all 3 orifice plate designs, suggests that there is an anti-phase effect. Part of the overall synthetic jet noise is suppressed because the two orifices in the double-chamber configuration act as a dipole source. Thus the sound produced by the two exhaled jets have 180 degrees phase difference which partially cancel each other out. Moreover, the noise output does not correlate with the jet velocity.

The sound pressure level trend in all orifice designs is generally the same. The noise gradually increases as the excitation frequency is increased up to the point where the maximum noise output level is achieved $(2.0-2.2 \mathrm{kHz})$. Note that the frequency at which the SJA produces maximum SPL is the same for all orifice designs.

The double chamber SJA with circular orifices (Fig. 14a), has an average noise reduction of $9 \%$ across the range of frequencies tested. It can also be seen that the maximum noise output is approximately $70 \mathrm{dBA}$ and $61 \mathrm{dBA}$ for single and double chamber SJAs respectively at $2.0 \mathrm{kHz}$. The sound level steadily decreases for frequencies higher than the resonant frequency. Furthermore at the Helmholtz resonant frequency of $1.6 \mathrm{kHz}$ the noise reduction caused by anti-phase is $16 \%$, whereas at the mechanical resonance of the diaphragm the suppression is only $6 \%$.

For the low corrugated orifice (Fig. 14b), the average noise reduction is only 5\%. The maximum noise reduction of $18 \%$ is observed at $2.6 \mathrm{kHz}$, however the synthetic jet velocity at that frequency is very small (outside of the resonant frequencies). The maximum noise output of the low corrugated orifice SJA is observed at $2.1 \mathrm{kHz}$ at $\sim 69$ $\mathrm{dBA}$ and $\sim 64 \mathrm{dBA}$ for single and double chamber respectively.

The average effect of anti-phase on the high amplitude corrugated-lobed orifice is a 5\% noise reduction (Fig. 14c). The maximum noise output of $\sim 68 \mathrm{dBA}$ is seen at $2.2 \mathrm{kHz}$ for both actuator configurations. The equivalent Helmholtz resonant frequency for the velocity experiment at $100 \mathrm{~V}_{p p}$ is between $1.5-1.6 \mathrm{kHz}$. At these frequencies, the noise difference between the single and double chamber actuator is around $15 \%$.

\section{Orifice shape comparisons}

The aim of this section is to evaluate the impact of the different orifice designs on the noise output of the doublechamber SJA. The three orifice plates generate noise gradually at low frequencies up to the point where the first resonant frequency occurs (Helmholtz Frequency - first vertical line on Fig. 15). Then there is a sudden increase of the sound pressure level until the second resonant frequency (natural frequency of the diaphragm - second vertical line on Fig. 15). It is observed that the circular orifice has a higher noise output than both corrugated-lobed orifices at frequencies ranging from $1.1-2.0 \mathrm{kHz}$. In contrast, at excitation frequencies higher than $2.0 \mathrm{kHz}$ the corrugatedlobed orifices are noisier than the circular orifice.

The maximum peak velocity of all three configurations is between 1.6 and $2.0 \mathrm{kHz}$ and thus the desired operating frequency of the actuator would also be within that range. In this range, the corrugated-lobed orifices produce lower noise output than the circular orifice. The maximum noise reduction is observed at $1.7 \mathrm{kHz}$ where the low corrugated orifice suppresses the sound by $24 \%$ and the high corrugated orifice by $26 \%$. At the point where the mechanical resonance of the diaphragm occurs (between $1.7-1.9 \mathrm{kHz}$ based on jet velocity results), the high corrugated-lobed orifice is the quietest, with sound pressure levels ranging from 47 to $57 \mathrm{dBA}$. However, the difference between the high and low corrugated orifices is only 2-3 dBA within that frequency region. It is believed that this is due to the lobes of both plates not being sufficiently distinctive, as identified in the manufacturing discrepancies. Therefore, the mixing enhancement of the turbulent jet flow with the ambient will be very similar.

To summarise, Fig. 16 presents a breakdown of the difference in sound pressure levels (in dBA) between the different SJA chamber configurations and orifice shapes analysed in this work. The SPL values correspond to the Helmholtz resonant excitation frequency. It can be seen that there is an overall SPL reduction of approximately 20 dBA from using a single-chamber, circular orifice SJA $(61 \mathrm{dBA})$ to a double-chamber, high-amplitude corrugated lobed orifice SJA (41 dBA). 
(a) SPL [dBA]

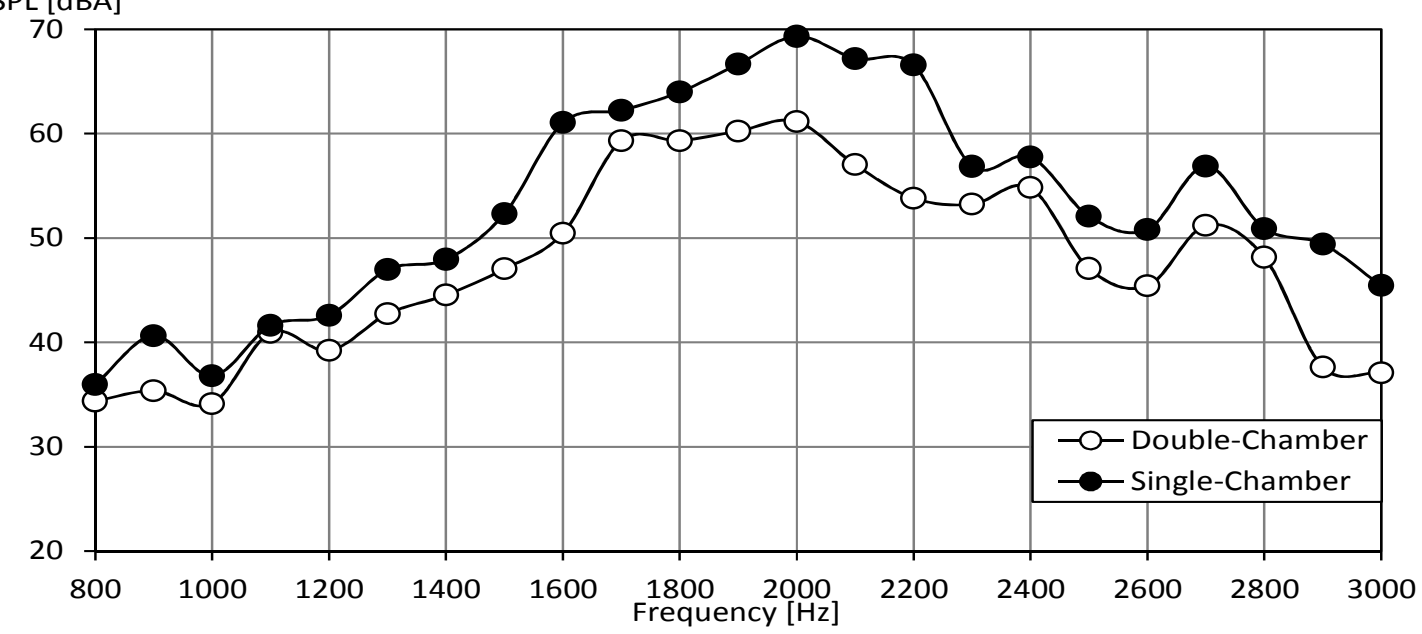

(b) SPL [dBA]

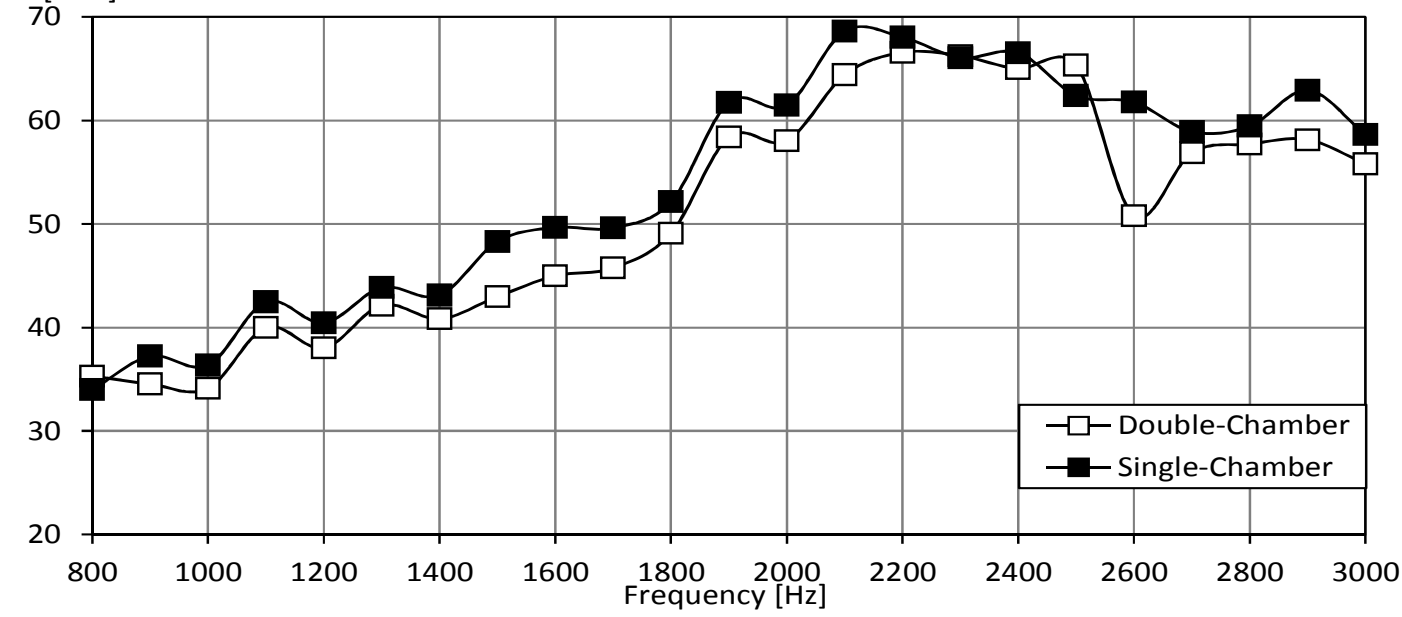

(c) SPL [dBA]

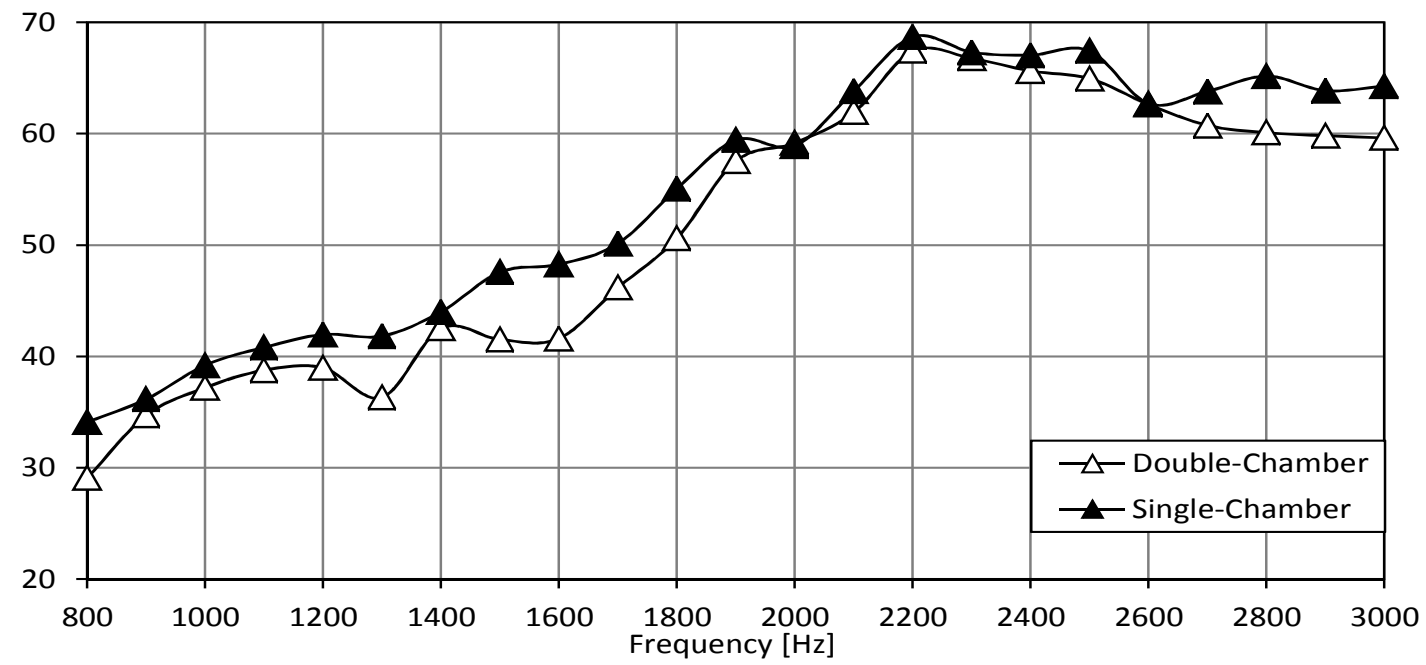

Figure 14. Noise output comparison between single- and double-chamber SJA configurations: (a) circular orifice; (b) low-amplitude corrugated lobed orifice; (c) high-amplitude corrugated lobed orifice

American Institute of Aeronautics and Astronautics 


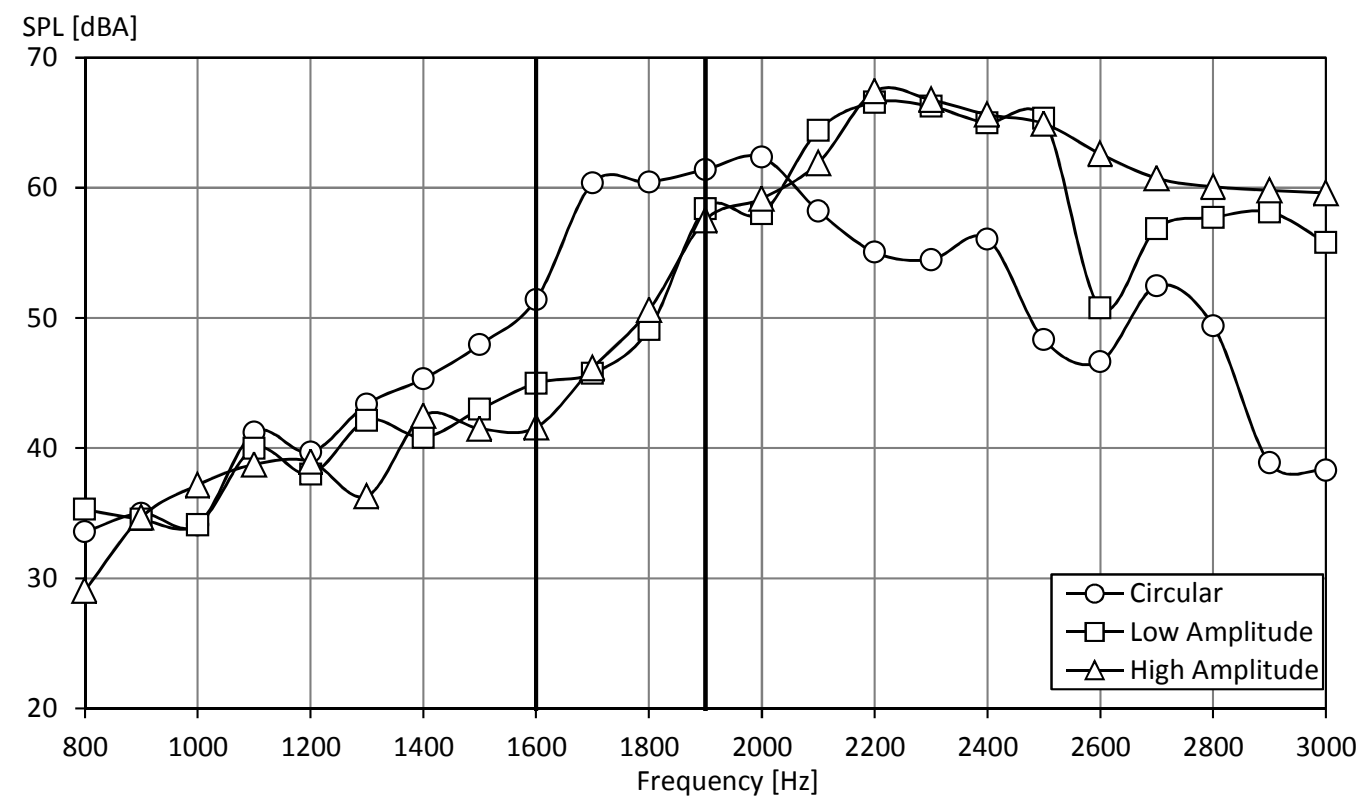

Figure 15. Comparison of jet noise output between circular, low corrugated and high corrugated orifice shapes in the double-chamber SJA configuration

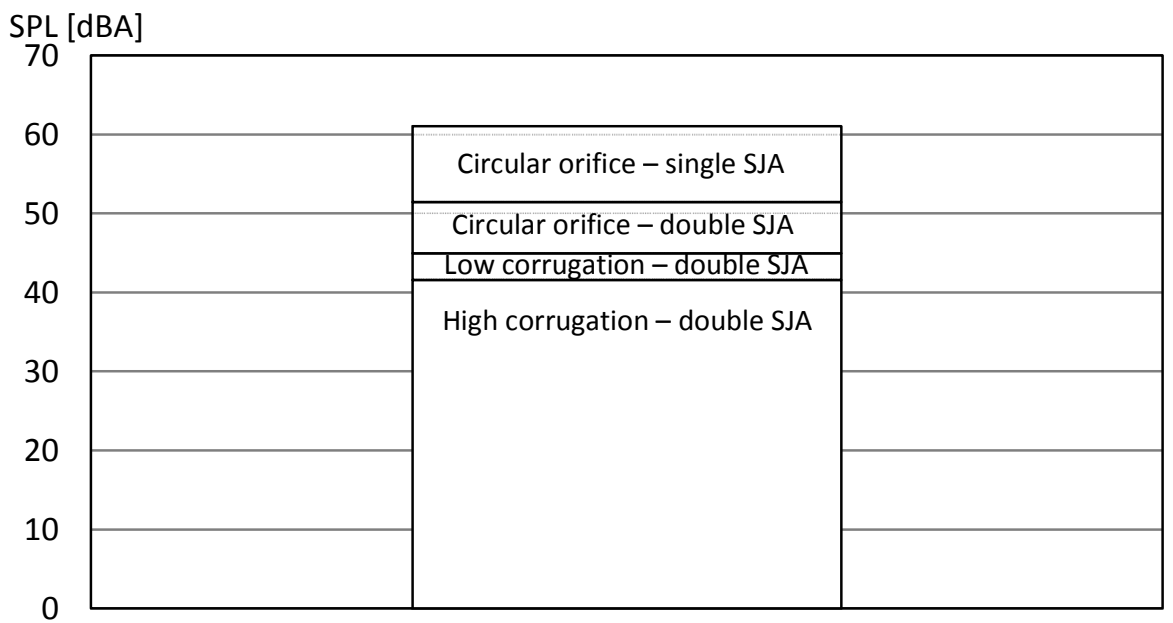

Figure 16. SPL breakdown of all SJA design variations at the Helmholtz resonant frequency

\section{Conclusions}

This paper has documented the design and development of piezoelectrical-driven synthetic jet actuator (SJA) hardware with the aim of delivering a reduction in overall SJA noise output while maintaining peak jet velocity output. Hardware development included the use of double-chamber or 'back-to-back' SJAs to reduce noise via 
dipole sources/anti-phase effect, and corrugated-lobed orifices to reduce noise via intensified jet flow mixing. The experimental work consisted of hot-wire anemometry and microphone recordings to conduct peak jet velocity and sound pressure level (SPL) measurements respectively of said SJA design variations. Measurements were compared with a baseline SJA (single chamber; circular orifice) across a range of actuator excitation frequencies $(800-3000$ $\mathrm{Hz})$ and for a fixed drive voltage $\left(100 \mathrm{~V}_{p p}\right)$.

Specific conclusions from the experimental work are as follows:

1. The use of a double-chamber, circular orifice SJA reduces actuator noise by up to $16 \%(10 \mathrm{dBA})$ at the resonant frequency $(\sim 1.6 \mathrm{kHz})$ compared with the baseline single-chamber SJA, which is simulated by blocking up one of the orifices of the double-chamber arrangement. The peak velocities of both single- and double-chamber SJAs are also the same, at approximately $37 \mathrm{~m} / \mathrm{s}$. The fact that the peak velocity is the same also illustrates that in the double-chamber configuration the two orifices, which are separated by a distance of 5 orifice diameters $(5 \mathrm{~mm})$, do not have fluid interaction with each other that causes jet velocity to be diminished.

2. The use of a corrugated-lobed orifice reduces actuator noise by up to $10 \mathrm{dBA}$ at the resonant frequency compared with a circular orifice of the same discharge area in the double-chamber configuration. The peak velocity of the high-amplitude, corrugated-lobed orifice SJA was reduced by $3 \mathrm{~m} / \mathrm{s}$ to $35 \mathrm{~m} / \mathrm{s}$, compared with the uncorrugated circular orifice.

3. The difference in noise output between the high-amplitude (lobe radius $=0.5 \mathrm{~mm}$ ) and low-amplitude (lobe radius $=0.25 \mathrm{~mm}$ ) corrugated orifices was less than $5 \mathrm{dBA}$, the low value of which is attributed to the identified manufacturing discrepancies.

4. An overall SPL reduction of $32 \%(20 \mathrm{dBA})$ was attained by switching from a single-chamber, circular orifice SJA (61 dBA) to a double-chamber, high-amplitude corrugated-lobed orifice SJA (41 dBA).

5. The electrical-to-fluidic power conversion efficiency of the double-chamber SJA is approximately $15 \%$ at the resonant frequency for all orifice types. This efficiency is double to that of the single-chamber SJA efficiency $(\sim 8 \%)$, due to the SJAs in the double-chamber configuration sharing the same piezoelectric disc with double the number of orifices each generating approximately the same fluid output power as a single-chamber SJA orifice.

This work has demonstrated feasible means by which the noise output of a SJA can be reduced substantially through chamber configuration and orifice shape.

\section{References}

${ }^{1}$ European Commision. (2011). Flightpath 2050: Europe's Vision for Aviation. Available online: http://ec.europa.eu/transport/modes/air/doc/flightpath2050.pdf. [Accessed: 02/05/2014].

${ }^{2}$ Arik, M. "An Investigation into Feasibility of Impingement Heat Transfer and Acoustic Abatement of Meso Scale Synthetic Jets," Applied Thermal Engineering, Vol. 27, 2007, pp. 1483-1494.

${ }^{3}$ Clarke, C. "Double-Chamber Synthetic Jet Actuators," Undergraduate Dissertation, School of Mechanical, Aerospace, Civil and Electrical Engineering, University of Manchester, UK, 2010.

${ }^{4}$ Anderson, D. F. and Bratos-Anderson, M. Noise: Its Measurement, Analysis, Rating and Control, Avebury Technical, $1^{\text {st }}$ ed., Hampshire, 1993.

${ }^{5}$ Lin, F., Schoen, M. P., Sekhri, R. and Ramkumar, B. "Modeling Acoustic/Structural Interaction of Synthetic Jet Actuators," Journal of Vibration and Control, Vol. 16, No. 9, 2010, pp. 1393-1414.

${ }^{6}$ Bies, D.A. and Hansen, C.H. Engineering Noise Control. Theory and Practice. E \& FN Spon, $2^{\text {nd }}$ ed., New York, 1996.

${ }^{7}$ Kopiev, V.F., Zaytsev, M. Y. and Ostrikov, N. N. "Subsonic Jet Noise Suppression by a Corrugated Nozzle," Acoustical Physics, Vol. 59, No. 2, 2013, pp. 207-209.

${ }^{8}$ Bargsten, C.J., and Gibson, M.T. "NASA Innovation in Aeronautics: Select Technologies That Have Shaped Modern Aviation," American Society for Engineering Education, NASA Technical Report, Washington DC, 2011.

${ }^{9}$ Tide, P. and Srinivasan, K. "Effect of Chevron Count and Penetration on the Acoustic Characteristics of Chevron Nozzles," Applied Acoustics, Vol. 71, 2010, pp. 201-220.

${ }^{10}$ Nastase, I. and Meslem, A. "Passive Control of Jet Flows Using Lobed Nozzle Geometries," Mécanique and Industries, Vol. 8, 2007, pp. 101-109.

${ }^{11}$ Norton, M. P. and Karczub, D.G. Fundamentals of Noise and Vibration Analysis for Engineers. Cambridge University Press, $2^{\text {nd }}$ ed., Cambridge, 2003.

${ }^{12}$ Crowther, W. J. and Gomes, L T. "An Evaluation of the Mass and Power Scaling of Synthetic Jet Actuator Flow Control Technology for Civil Transport Aircraft Applications," Proceedings of the IMechE. Part I: Journal of Systems and Control Engineering, Vol. 222, 2008, pp. 357-372.

${ }^{13}$ Jabbal, M., Liddle, S., Potts, J. and Crowther, W. "Development of Design Methodology for a Synthetic Jet Actuator Array for Flow Separation Control Applications," Proceedings of the IMechE. Part G: Journal of Aerospace Engineering, Vol. 227, No. 1, 2013, pp. 110-124. 
${ }^{14}$ Jabbal, M., Liddle, S.C. and Crowther, W.J. "Active Flow Control Systems Architectures for Civil Transport Aircraft," Journal of Aircraft, Vol. 47, No. 6, 2010, pp. 1966-1981.

${ }^{15}$ Gomes, L.D., Crowther, W.J. and Wood, N.J. "Towards a Practical Piezoceramic Diaphragm Based Synthetic Jet Actuator for High Subsonic Applications - Effect of Chamber and Orifice Depth on Actuator Peak Velocity," Proceedings of the $3^{\text {rd }}$ AIAA Flow Control Conference, AIAA 2006-2859, San Francisco, CA, 5-8 June 2006.

${ }^{16}$ Jain, M., Puranik, B. and Agrawal, A. "A Numerical Investigation of Effects of Cavity and Orifice Parameters on the Characteristics of a Synthetic Jet Flow," Sensors and Actuators A: Physical, Vol. 165, 2011, pp. 351-366.

${ }^{17}$ Godard, G. and Stanislas, M. "Control of a Decelerating Boundary Layer. Part 1: Optimisation of Passive Vortex Generators," Aerospace, Science and Technology, Vol. 10, No. 3, 2006, pp. 181-191.

${ }^{18}$ Godard, G. and Stanislas, M. "Control of a Decelerating Boundary Layer. Part 3: Optimisation of Round Jets Vortex Generators," Aerospace, Science and Technology, Vol. 10, No. 6, 2006, pp. 455-464.

${ }^{19}$ Crowther, W.J. "Control of Separation on a Trailing Edge Flap Using Air Jet Vortex Generators," Journal of Aircraft, Vol. 43, No. 5, 2006, pp. 1589-1593.

${ }^{20}$ Flynn, K.P. and Panton, R.L. "The Interaction of Helmholtz Resonators in a Row When Excited by a Turbulent Boundary Layer," Journal of Acoutical Society America, Vol. 87, No. 4, 1990, pp. 1482-1488.

${ }^{21}$ Gallas, Q., Holman, R., Nishida, T. "Lumped Element Modeling of Piezoelectrical-Driven Synthetic Jet Actuators," AIAA Journal, Vol. 41, No. 2, 2003, pp. 240-247.

${ }^{22}$ Belvins, R.D. Formulas for Natural Frequency and Mode Shape, Von Nostrand Reinhold Company, $2^{\text {nd }}$ ed., New York, 1979.

${ }^{23} \mathrm{Li}$, R., Sharma, R. and Arik, M. "Energy Conversion Efficiency of Synthetic Jets," Proceedings of the ASME Pacific Rim Technical Conference and Exhibition on Packaging and Integration of Electronics and Photonic Systems, MEMS and NEMS, Vol. InterPACK 2011, pp. 1-8. 